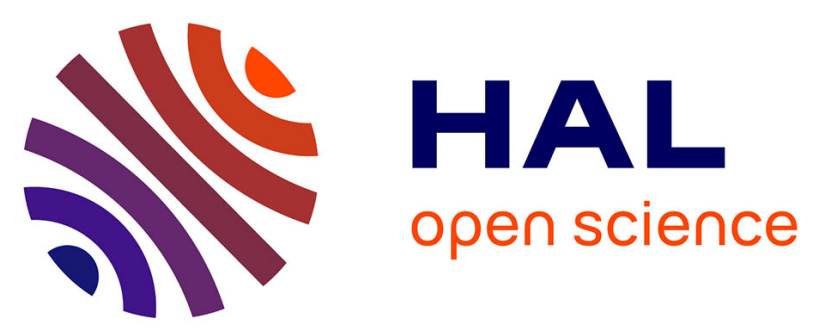

\title{
Osteogenic potential of adipogenic predifferentiated human bone marrow-derived multipotent stromal cells for bone tissue-engineering
}

\author{
Adrien Moya, Nathanael Larochette, Marianne Bourguignon, Hanane \\ El-Hafci, Esther Potier, Hervé Petite, Delphine Logeart-Avramoglou
}

\section{To cite this version:}

Adrien Moya, Nathanael Larochette, Marianne Bourguignon, Hanane El-Hafci, Esther Potier, et al.. Osteogenic potential of adipogenic predifferentiated human bone marrow-derived multipotent stromal cells for bone tissue-engineering. Journal of Tissue Engineering and Regenerative Medicine, 2018, 12 (3), 10.1002/term.2571 . hal-02368382

\section{HAL Id: hal-02368382 \\ https://hal-univ-paris.archives-ouvertes.fr/hal-02368382}

Submitted on 1 Dec 2020

HAL is a multi-disciplinary open access archive for the deposit and dissemination of scientific research documents, whether they are published or not. The documents may come from teaching and research institutions in France or abroad, or from public or private research centers.
L'archive ouverte pluridisciplinaire HAL, est destinée au dépôt et à la diffusion de documents scientifiques de niveau recherche, publiés ou non, émanant des établissements d'enseignement et de recherche français ou étrangers, des laboratoires publics ou privés. 
Moya et al.

\title{
Osteogenic potential of adipogenic predifferentiated human bone marrow-derived multipotent stromal cells for bone tissue- engineering
}

\author{
Authors : Adrien Moya ${ }^{1}$, Nathanaël Larochette ${ }^{1}$, Marianne Bourguignon ${ }^{1}$, Hanane El-Hafci ${ }^{1}$, Esther Potier \\ 1, Hervé Petite ${ }^{1}$, Delphine Logeart-Avramoglou ${ }^{1}$
}

\begin{abstract}
Affiliations:
1: Laboratory of Bioengineering and Bioimaging for Osteo-Articular tissues UMR 7052 CNRS, Univ Paris Diderot, Sorbonne Paris Cité, France
\end{abstract}

Key words: adipogenesis; bone; MSC; osteogen esis; predifferentiation; tissue-engineering

\begin{abstract}
In the present study, we evaluated the benefits of an adipogenic predifferentiation, the pathway most closely related to osteoblastogenesis, on the pro-osteogenic potential of human adult multipotent bone marrow stromal cells (hBMSCs), both in vitro and in vivo. Adipogenic differentiation of hBMSCs for 14 days resulted in a heterogeneous cell population from which the most adipogenic-committed cells were eliminated by their lack of readhesion ability. Our results provided evidence that the select adherent adipogenic differentiated hBMSCs (sAD+ cells) express a gene profile characteristic of both adipogenic and osteogenic lineages. In vitro, when cultured in osteogenic medium, sAD+ differentiated along the osteogenic lineage faster than undifferentiated hBMSCs. In vivo, in an ectopic mouse model, sAD+ exhibited a significantly higher bone formation capability compared with undifferentiated hBMSCs. We sought, then, to investigate the underlying mechanisms responsible for such beneficial effects of adipogenic predifferentiation on bone formation and found that this outcome was not linked to a better cell survival post-implantation. The secretome of SAD+ was both proangiogenic and chemoattractant, but its potential did not supersede the one of undifferentiated hBMSCs. However, using co-culture systems, we observed that the sAD+ paracrine factors were pro-osteogenic on undifferentiated hBMSCs. In conclusion, adipogenic priming endows hBMSCs with high osteogenic potential as well as pro-osteogenic paracrine-mediated activity. This preconditioning appears as a promising strategy for bone tissue engineering technology in order to improve the hBMSC osteogenic potency in vivo.
\end{abstract}

\section{INTRODUCTION:}

Adult bone marrow multipotent stromal cells/mesenchymal stem cells (BMSCs) have the potential to give rise to several tissues, including bone, cartilage, adipose, and muscle (Pittenger et al., 1999). Because of their osteogenic potential, BMSCs are one of the most promising stem cell populations for bone regeneration. Proof of efficacy for the strategy of using BMSC-seeded osteoconductive scaffolds has been established in clinically relevant large animal models (Bruder et al., 1998; Petite et al., 2000). To date, however, the outcomes of these studies are not as good as those using autologous bone grafts, which are the current "gold standard" in bone repair. This evidence emphasizes the great need for improving the efficacy of bone tissue-engineered constructs. Selection of the best material support scaffold, as well as determination of the optimal preparation of cells before implantation, are mandatory for future clinical translation.

For this reason, several cell priming approaches (including cell pre-differentiation prior to implantation) have been explored. Some research groups investigated whether an in vitro osteogenic induction of BMSCs could either facilitate or speed up subsequent bone formation in vivo. In general, however, such approaches yielded contradictory and disappointing results. Some studies reported that the osteogenic commitment of BMSCs prior to cell implantation was either ineffective in terms of improved ectopic bone formation (Janicki et al., 2011; Kasten et al., 2005; Niemeyer et al., 2006) or less effective than undifferentiated BMSCs in repairing rat calvarial bone defects (Zong et al., 2010); in contrast, other studies showed that osteogenic induction of BMSCs was beneficial and even essential for de novo bone formation (Vaquette, Ivanovski, Hamlet, \& Hutmacher, 2013; Ye, Yin, Yang, Tan, \& Liu, 2012). Aiming at triggering the endochondral ossification, other groups assessed the effect of in vitro chondrogenic priming of BMSCs onto in vivo osteogenesis. Pertinent results provided evidence that the chondrogenic priming of human BMSCs (hBMSCs) either in pellet (Pelttari et al., 2006) or seeded in hydrogels (Dickhut, Gottwald, Steck, Heisel, \& Richter, 2008) or on HA/TCP particles (Janicki et al., 2010; Jukes et al., 2008) had no or little success in inducing heterotopic bone formation in mice.

Among the possible differentiation outcomes of BMSCs, the adipogenic and osteogenic pathways are the most closely related and play an important role in regulating bone mass homeostasis (reviewed in Muruganandan, Roman, \& Sinal, 
Moya et al.

2009). There is a growing body of evidence in the scientific literature that, given their proximity in the bone marrow milieu, a "dialogue" exists between adipocytes, osteoblasts, and BMSCs through several soluble factors. In addition, the molecular pathways governing hBMSC adipogenic and osteogenic differentiation appear interconnected. In vitro studies indicate that, based on lineage specific marker expression, there is a high degree of plasticity between the two lineages (Kollmer, Buhrman, Zhang, \& Gemeinhart, 2013; Schilling, Noth, Klein-Hitpass, Jakob, \& Schutze, 2007); coexpression of osteoblastic and adipogenic markers were also observed in hBMSC-derived osteoblasts at the single-cell-level (Ponce et al., 2008). These observations suggest that the osteoblastic and adipogenic differentiations in hBMSCs are not only competing and reciprocal but also possibly occur in parallel until a late stage during the osteoblastic differentiation process.

The present study demonstrated for the first time that preinduction of hBMSCs towards the adipogenic lineage enhances their bone forming potency. The hBMSC-derived preadipocytes used expressed both adipogenic and osteogenic markers and differentiated along the osteogenic lineage faster than unprimed hBMSCs. The underlying mechanisms of such effects was further investigated by assessing select aspects of the adipogenic-primed hBMSC secretome (including the proangiogenic, chemoattractive, and pro-osteogenic potentials) pertinent to bone tissue formation.

\section{Materials and Methods:}

\subsection{Cells and cell cultures}

\subsubsection{Cell isolation}

hBMSCs were harvested from bone marrow obtained as discarded tissue during routine bone surgery from five adult donors at the Lariboisiere Hospital (Paris, France). The tissues were collected with the respective donor's consent in agreement with Lariboisiere Hospital regulations. hBMSCs were isolated from each donor's bone marrow using a procedure adapted from literature reports (Friedenstein, Chailakhjan, \& Lalykina, 1970) and were characterized for their expression of select CD markers (CD90, CD73, CD105, and CD45; Figure S1) and for their cell differentiation potential along either osteogenic, adipogenic, or chondrogenic lineages (Figure S2). All experiments described in this manuscript were performed using these hBMSCs pooled at an equal ratio at passage 1, except for a set of in vivo experiments where hBMSCs from three donors were used separately. hBMSCs were cultured in Alpha Minimum Essential Medium ( $\alpha \mathrm{MEM}$; Sigma) supplemented with $10 \%$ foetal bovine serum (FBS; PAA Laboratories) and antibiotics (referred as standard culture medium) in a humidified, $37{ }^{\circ} \mathrm{C}, 5 \% \mathrm{CO} 2$ balance air environment (referred as standard culture conditions). hBMSCs up to passage 6 were used for the experiments.

\subsubsection{Cell culture under ischaemic and hypoxic conditions}

For cell culture under ischaemic conditions, hBMSCs were seeded at 12,000 cells/cm2 in 24 well plates and cultured for 14 consecutive days in serum- and glucose-free $\alpha$ MEM (PAN Biotech). These cells were placed in a proOx-C-chamber system
(C-Chamber, C-374; Biospherix), in which the oxygen concentration was maintained at $0.1 \%$ for the duration of pertinent experiments. For cell culture under hypoxic conditions, the protocol was the same as that used for the experiments under ischaemic conditions, except that the cells were cultured in serum-free $\alpha$ MEM supplemented with $(5 \mathrm{~g} / \mathrm{L}$ ) glucose for seven consecutive days. At that time, the supernatants (conditioned media) were collected and stored at $-80{ }^{\circ} \mathrm{C}$ until further analysis. In order to ensure constant oxygen levels, the cell cultures were maintained undisturbed and without medium change until the end of the experiments.

\subsubsection{Adipogenic and osteogenic predifferentiation / differentiation}

hBMSCs were seeded at 12,000 cells $/ \mathrm{cm} 2$ and cultured in adipogenic medium (containing 10-7-M dexamethasone, 0.5$\mathrm{mM}$ IsoButylMethylXanthine, $60-\mu \mathrm{M}$ indomethacine, and 5$\mu \mathrm{g} / \mathrm{ml}$ insulin; all chemicals from Sigma-Aldrich) for 14 days; these cells will be referred to as "hBMSCs-Adt" for the rest of the manuscript. At that time, the cells were trypsinized and reseeded onto tissue-culture plasticware (for in vitro studies) or onto coral particles (for in vivo studies); these cells will be referred to as "sAD+ cells" hereafter. Lipid accumulation was evaluated in hBMSCs during their adipogenic differentiation for up to 21 days of culture using a technique described by Lee, Chen, Wiesner, and Huang (2004). Briefly, the cells were rinsed with PBS, trypsinized, and analysed using an Attune ${ }^{\circledR}$ flow Cytometer (Life Technologies). The lipid-containing cells possessed increased granularity that were detected in the side scatter axis. The number of lipid-vesicles containing-cells was expressed as a percentage of the total cell population. In some experiments, sAD+ cells were compared with osteogenic predifferentiated hBMSCs, which were prepared by culturing 12,000 cells $/ \mathrm{cm} 2$ in osteogenic medium (containing 10-7-M dexamethasone, 150- $\mu \mathrm{M}$ ascorbic acid-2 phosphate, and 2-mM beta glycerophosphate) for 14 days, trypsinized and reseeded (referred to as "sOS+ cells" hereafter). For osteogenic differentiation, either sAD+ cells, hBMSCs, or sOS+ cells (serving as control cells) were seeded at 12,000 cells/cm2 and were further cultured in osteogenic medium.

\subsection{Osteogenic and ad ipogenic differentiation analyses}

After 7 and 14 days of culture in osteogenic medium, the alkaline phosphatase (ALP) activity in cell lysates was quantified as previously described (Becquart et al., 2012). After 14, 21, and 28 days of culture in osteogenic medium, the presence of calcium-containing deposits in the extracellular matrix was determined using Alizarin Red stain and visualized using light microscopy. The alizarin red stain was extracted, and its optical density was measured at $410 \mathrm{~nm}$. After 0,14 , and 21 days of culture in adipogenic medium, lipid accumulation was assessed by staining the cells using Oil Red 0 stain, and visualizing lipid-containing droplets using light microscopy. Real-time quantitative polymerase chain reactions (RT-qPCRs) targeting the genes implicated in either adipogenesis or osteogenesis were also performed. Cells were rinsed with PBS, and total RNA was extracted using Trizol®. The RNA concentration and purity were determined using a NanoDrop spectrophotometer (NanoDrop 1000, Labtech). 
Moya et al.

cDNA was obtained after reverse transcription of $1.5 \mu \mathrm{g}$ of purified RNA using the superscript II enzyme (Life Technologies) and random primers. Then, quantitative PCR was performed using 25-ng cDNA in 96 well plates (iCycler iQ PCR plates; Biorad) using Taqman gene expression assays (Life Technologies) for PPAR $\gamma$, CEBPA, LEP, LPL, GLUT4, ALP, RUNX2, IBSP, and bGLAP following the manufacturer's instructions and using the $\mathrm{MyiQ}^{\mathrm{TM}}$ Single-Colour Real-Time PCR Detection System (Biorad). For all experiments, the results were normalized first to that of the respective 18S (internal standard), and then to results obtained for hBMSCs at Day 0. The full names of the genes monitored and assay IDs are given in Table S1.

\subsection{Quantification of viable cells in vitro.}

Viable cells were identified after treatment with both 0.5$\mu \mathrm{g} / \mathrm{ml}$ propidium iodide and $1-\mu \mathrm{g} / \mathrm{ml}$ Hoechst 33342 (Sigma) at $37^{\circ} \mathrm{C}$ for $30 \mathrm{~min}$. The labelled cells were washed with PBS, trypsinized, and analysed using flow cytometry. Cells which had been stained both Hoechst positive and propidium iodide negative were identified as "viable cells." At each prescribed time point, cell viability was expressed as the number of viable cells normalized by the respective viable cell number at Day 0 (D0).

\subsection{Assessment of the angiogenic effect of supernatant conditioned medium}

Formation of vascular-like structures were observed $20 \mathrm{hr}$ after seeding 10,000 human umbilical vein endothelial cells (HUVEC; purchased from Lonza)/cm2 on the top surface of Matrigel hydrogels (Matrigel Growth Factor Reduced, BD Falcon; BD Biosciences) and covered with $500 \mu \mathrm{l}$ of conditioned media from either hBMSCs or sAD+ cells. The vascular-like structures in all Matrigels were then observed using light microscopy, photographed, and counted. $\alpha \mathrm{MEM}$ containing $10 \%$ FBS and ( $1 \mathrm{~g} / \mathrm{L})$ glucose ( $\alpha$ MEM-10\% FBS) was the positive control medium while serum- and glucosefree $\alpha$ MEM medium was the negative control medium ( $\alpha$ MEM$0 \%$ FBS).

\subsection{Chemotactic cell migration in Boyden chambers}

The chemoattractive potential of conditioned media was determined using the Boyden chamber migration assay. Briefly, $600 \mu \mathrm{l}$ of each respective conditioned medium were added in the bottom well, and hBMSCs were seeded on the top of a porous $(8-\mu \mathrm{m}$ pore-diameter) membrane (previously coated with $0.5 \%$ gelatin) of Transwell (Corning® Costar $\AA$ Transwell@ chambers). After $6 \mathrm{hr}$, the migrated hBMSCs were fixed in situ using 11\% glutaraldehyde (Fluka BioChemika), stained using the modified Giemsa stain (Sigma-Aldrich), and counted following visualization using light microscopy. Cells that did not migrate were removed by scrapping the top of the porous Transwell membrane. $\alpha$ MEM- $10 \%$ FBS was the positive control medium, and $\alpha \mathrm{MEM}-0 \%$ FBS was the negative control medium.

\subsection{Co-cultures without direct cell-cell contact}

hBMSCs were seeded at a density of 12,000 cells $/ \mathrm{cm} 2$ into individual wells of 12 well plates. Either SAD+ or hBMSCs were seeded at the same density into cell-culture-inserts (with 0.4- $\mu \mathrm{m}$ pore diameter; Millicell), which were then transferred into the wells with seeded hBMSC. Co-cultured cells were maintained under standard culture conditions in osteogenic medium for up to 21 days.

\subsection{In vivo studies}

\subsubsection{Preparation of cell-containing constructs}

Forty milligrams of coral ceramic granules (80-200 $\mu \mathrm{m}$; Biocoral ${ }^{\circledR}$, Inoteb, Inc.) were autoclaved and washed using standard culture medium for $1 \mathrm{hr}$. After medium removal, either hBMSCs or sAD+ cells (106 cells in $400 \mu \mathrm{L}$ of $\alpha \mathrm{MEM}$ $10 \%$ FBS) were seeded onto the coral particles and allowed to adhere at $37^{\circ} \mathrm{C}$ overnight. Prior to implantation, the culture medium was discarded and the cell-seeded granules were embedded in 100- $\mu$ l fibrin gel $(18-\mathrm{mg} / \mathrm{ml}$ fibrinogen; $65-\mathrm{U} / \mathrm{ml}$ Thrombin, Tissucol@; Baxter).

\subsubsection{In vivo implantation}

Eight-week-old female nude mice were obtained from JanvierLabs and handled in accordance with the European Directive 2010/63/EU regarding the protection of animals used for scientific purposes. All animal experiments were performed following approval of the pertinent protocols by the Ethics Committee on Animal Research of Lariboisiere/Villemin (number CEEA-LV/2014-10-20) Paris, France. Cell-containing constructs were subcutaneously implanted in mice as previously described (Becquart et al., 2012).

\subsubsection{In vivo cell survival analysis}

hBMSCs were previously genetically modified by their transduction using a lentiviral vector encoding firefly luciferase (pRRLsin-MND-Luc-IRES2-ZsGreen-WPRE; TBMCore platform; Bordeaux; Becquart et al., 2012) and further expanded. Cell viability in both hBMSC(Luc) and sAD+(Luc) cell-containing constructs ( $\mathrm{n}=6$ constructs per group) was monitored non-invasively in vivo using a bioluminescence imaging system (Ivis, Lumina II®, Caliper Life Sciences) as previously described (Logeart-Avramoglou et al., 2010) throughout the 30 days of implantation. Results were expressed as a percentage of the bioluminescence signal measured at D1 post-implantation.

\subsection{Histology, histomorphometry, and immunohistology}

After 8 weeks of implantation, the mice were sacrificed (using Dolethal $($; Vetoquinol) and the retrieved constructs ( $\mathrm{n}=11$ and $\mathrm{n}=14$ for $\mathrm{sAD}+$ cell and undifferentiated hBMSC groups, respectively) were processed for undecalcified histology and histomorphometric analysis as described previously (Monfoulet et al., 2014). The surface area of bone (stained in red) was measured in each specimen section and normalized over the whole surface area delineated around each section. Chemical composition analysis of either mouse cortical bone or cell-construct bone was performed using backscattered scanning electron microscopy and image analysis using an Analytical UHR Schottky Emission Scanning Electron Microscope SU-70 (HITACHI).

After 8 weeks of implantation, the luciferase-labelled cell constructs were retrieved ( $n=6$ per group), fixed in $4 \%$ paraformaldehyde (pH 7.4), decalcified in 
Moya et al.

ethylenediaminetetraacetic acid $(14.5 \% \mathrm{w} / \mathrm{v})$ at $4{ }^{\circ} \mathrm{C}$ for 2 weeks, and embedded in paraffin. Some sequential sections were stained with Masson's Trichrome. The number of blood vessels per section was counted for all explants using the ImageJ software (ImageJ; NIH, Bethesda, Maryland, USA). Some other sequential sections were processed for human beta-2microglobulin (a membrane protein that enables tracking human cells) immunodetection, using the Envision + Kit (Dako) and polyclonal rabbit anti-beta-2-microglobulin (1/1000; Novocastra) as the primary antibody (Monfoulet et al., 2014).

\subsection{Statistical analyses}

Numerical data were expressed as mean \pm standard deviation $( \pm$ SD). Statistical analyses were conducted using the Statgraphics centurion version XV.2 (Statpoint, Inc.) software. The nonparametric Mann-Whitney U test was used to analyze data from two independent samples. The one-way ANOVA followed by Tukey's post-hoc test was used to compare means of three or more groups. The quantitative kinetics data were analysed using two-way ANOVA followed by Tukey's post-hoc test. For all analyses, differences at $\mathrm{p}<.05$ were considered as statistically significant.In vivo studies

\section{RESULTS}

3.1 Select adipogenic preconditioned hBMSCs (sAD+) share gene markers of both adipogenic and osteoblastic lineage

Adipogenic differentiation of hBMSCs after 14 days of culture resulted in a highly heterogeneous cell population (referred to as "hBMSC-Ad+" hereafter) containing various cells ranging from lipid-free hBMSCs to mature adipocytes filled with lipid vesicles (Figure 1a, 1b, 1cA, and $1 \mathrm{cB}$ ). The percentage of hBMSCs containing lipid vesicles increased with the duration of the cell culture period in the adipogenic medium and reached $50 \%$ and $65 \%$ of the total cell population after 14 and 21 days of culture, respectively (Figure 1b). Trypsinization and reseeding of hBMSC-Ad+ onto tissue culture plasticware resulted in elimination of hBMSCs fully filled with lipid vesicles (considered as the most mature adipocytes); these cells did not readhere on substrate surfaces. The adherent cell population (sAD+ cells) contained $88 \%$ of lipid-free hBMSCs and $12 \%$ of lipid-containing hBMSCs; they also displayed a spindle-shaped morphology typical of the undifferentiated hBMSCs (Figure 1cC and $1 \mathrm{cD})$..

The sAD+ cells were further characterized 24-hr postreseeding by assessing expression of a set of surface antigens. Compared with the undifferentiated hBMSCs, the sAD+ cells maintained expression of CD73, CD90, and CD105 (although reduced by $42 \%$ and $32 \%$ for the $\mathrm{CD} 90$ and $\mathrm{CD} 105$, respectively). In comparison, unselected hBMSC-Ad+ cell populations also remained positive for CD73 and exhibited a $67 \%$ reduction of CD90 expression but lost the CD105 marker (Figure S1). Expression for CD45 was negative in all cell populations tested.

Analysis of the expressed adipogenesis-related genes showed that, compared with undifferentiated hBMSCs, the two transcription factors Peroxisome proliferator-activated receptor gamma (PPAR $\gamma$ ) and CCAAT/Enhancer Binding Protein (C/EBP- $\beta$ ), as well as the Leptin (LEP), lipoprotein lipase (LPL) genes were all significantly upregulated in SAD+ cells (24-hr post-seeding); in contrast, the Glucose Transporter Type 4 (Glut4) gene was downregulated (Figure 1d). These genes, however, were expressed at a lesser extent in the sAD+ than in the nonselected hBMSC-Ad+ cell populations (Figure 1d).

Analysis of the expressed osteogenesis-related genes in these cell populations revealed that, in comparison with undifferentiated hBMSCs and hBMSC-Ad+ cells, gene expression of the transcription factor Runt-related transcription factor 2 (RUNX2) was the same in all groups tested, both the Alkaline Phosphatase (ALP) and IntegrinBinding Sialoprotein (IBSP; two early osteogenic markers) gene expression levels were upregulated in the sAD+ (4.3- and 23 -fold, respectively; Figure 1e). In addition, Bone GammaCarboxyglutamate Protein (BGLAP, a late osteogenic marker) expression was similar to that obtained from the undifferentiated hBMSCs (Figure 1e).

To further examine the phenotype of the sAD+ cell population, their gene expressions were compared with those of select osteogenic predifferentiated hBMSC (sOS+). The gene expression of adipogenic-related genes was higher in the SAD+ cells than in sOS+ cells, with the exception of the expression of LEP, which was downregulated in the SAD+ compared with sOS+ cells. This latter result may be explained as the effect of dexamethasone (present in both adipogenic and osteogenic medium used in the present study) that was shown to upregulate LEP (Ghali et al., 2015). Interestingly, sAD+ expressed the osteogenic-related genes at the same (RUNX2, ALP, and bGLAP) level as or higher (IBSP) than the sOS+ cells (Figure 1e).

Because sAD+ cells are a heterogen eous cell population, singlecell analyses on sAD+ cells (in comparison with hBMSCs, hBMSC-Ad+, and sOS+ cell populations) were also performed by monitoring Bodipy (a lipid marker) positive cells (Bodipy $(+)$ ) and ALP positive cells (ALP $(+)$ ), and using flow cytometry (Figure S3). Results provided evidence that $\sim 8 \%$ of sAD+ cells were Bodipy(+) and that both Bodipy(+) and Bodipy $(-)$ cell subpopulations contained $45 \%$ and $57 \%$ $\mathrm{ALP}(+)$ cells, respectively, proving that the portion of cells expressing ALP was similar $(\sim 50 \%)$ in both lipid-free sAD+ and lipid-containing sAD+ cell populations (Figure S3). Besides, the selection step on hBMSC-Ad + cell enable to enrich ALP $(+)$ cells in the sAD+ cell population (from $47 \%$ to $57 \%$ and from $27 \%$ to $45 \%$ for Bodipy(+) and Bodipy(-) cell subpopulations, respectively; Figure S3).

\subsection{The SAD+ cells exhibit higher osteogenic potential than unpredifferentiated hBMSCs both in vitro and in vivo}

Whereas RUNX2 expression in control hBMSCs increased with the osteogenic culture period, its level was not modified in sAD+ (Figure 2a). Expression of both ALP and IBSP in sAD+ cells slightly decreased during the same culture period but remained higher than that observed in the hBMSCs. Similarly to the results obtained with the control group, BGLAP 
Moya et al.

expression increased with time in the sAD+ cells; the expression levels were similar for both groups (Figure 2a). With the exception of the IBSP gene (which was higher expressed in SAD+ than in sOS+ cells at D0 and D7), the levels of expression of these osteogenic-related genes in SAD+ were similar to those expressed in SOS+ (Figure 2a).

Upon osteogenic differentiation, only PPAR $\gamma$ and C/EBP- $\beta$ genes remained higher expressed in the $\mathrm{SAD}+$ cell group compared with results obtained from the hBMSC and sOS+ cell group (Figure S4)

The ALP activity in the three cell groups tested increased with the osteogenic culture period (Figure 2b). The ALP activity in the sAD+ cells was significantly higher than that observed in the hBMSCs but was significantly lower than in sOS+ cells at Days 0 and 7 of osteogenic differentiation; the ALP activity in the three cell groups was similar at Day 14 (Figure 2b). Extracellular matrix mineralization (assessed using Alizarin Red staining) was detected as early as Day 14 of osteogenic differentiation in the sAD+ cells but it was only detected at Day 28 in the hBMSCs (Figure 2c). Of note, lipid droplets in the sAD+ cell population were still visible after 21 days of osteogenic differentiation (arrow; Figure $2 \mathrm{cH}$ and 2cK). Absorbance of the eluted Alizarin red stain indicated that the sAD+ cells contained approximately 2.7-, 70-, and 1.3-fold greater values than hBMSC control cells after 14, 21, and 28 days of culture, respectively (Figure 2d). As can be expected from their pre-adipogenic phenotype, the sAD+ cells also displayed differentiation along the adipogenic lineage pathway much faster than the hBMSCs; the number of lipid-droplet containing cells was greater after both 14 and 21 days of adipogenic differentiation (Figure 2e).

De novo bone formation induced by sAD+ cells and undifferentiated hBMSCs after 8 weeks of ectopic implantation of cell-containing constructs (prepared with coral particles) in mice was assessed. Histological analyses showed that bone formation occurred in both cell groups was mainly located in the centre of the implant and surrounded the coral particles (Figure $3 \mathrm{aA}$ and $\mathrm{3aB}$ ). In contrast to bone tissue observed in all sAD+-containing constructs (bone incidence of 11/11), it was detected in only $6 / 14$ of the hBMSC-containing constructs (Figure 3b). Furthermore, the amount of deposited new bone tissue in the $\mathrm{SAD}+$ cell-containing constructs was 5-fold higher than in the hBMSC-containing constructs (Figure 3b). Backscattered scanning electron microscopy analyses performed on the bone formed in the sAD+ cell-containing constructs revealed that its composition was close, but not similar, to the mature murine cortical bone tissue, indicating that this newly formed bone tissue was still immature (Figure 3c-d).

To further confirm the in vivo pro-osteogenic potential of sAD+ cells and to ensure that this effect was not donor dependent, cell constructs containing either sAD+ or hBMSC cells from three separate donors were prepared and implanted ectopically for 6 and 10 weeks (Figure S5a-d). After 6 weeks of implantation, newly formed bone was only detected in constructs containing the sAD+ cells (bone incidence of 6/9 implants; Figure S5b). After 10 weeks of implantation, the bone incidence in implanted constructs was $8 / 9$ versus $3 / 9$ with the $\mathrm{sAD}+$ cells and hBMSCs, respectively (Figure S5d). These data confirmed that the enhanced osteogenic potential of the SAD+ cells was not donor dependent.
3.3 Enhanced bone formation induced by sAD+ cells is not related to improved cell survival under ischaemic conditions

The question of whether an improved survival of the sAD+ cells in an ischaemic environment explains their "superior" osteogenic potential was addressed. Cell viability was monitored in vitro under ischaemic conditions mimicking the conditions encountered by implanted cells, as well as in vivo after ectopic implantation of constructs containing cells previously labelled with the luciferase reporter gene and using bioluminescence imaging. Under in vitro ischaemic conditions (near-anoxia $(0.1 \% 02)$, as well as total glucose- and serumdepletion), the cells from both groups tested died at the same rate over the 14 days of culture (Figure 4a). In vivo, the bioluminescence signal from constructs containing either $\mathrm{sAD}+(\mathrm{Luc})$ cells or hBMSCs(Luc) rapidly decreased over time after subcutaneous implantation, indicating the disappearance of viable implanted cells (Figure $4 \mathrm{~b}$ ). The decreased sAD+(Luc) cell survival rate was even faster, but not significantly, than the one observed with the hBMSCs(Luc). After 30 days of implantation, the cell survival rates were low but not null for both groups (specifically, $15 \%$ and $24 \%$ for the sAD+(Luc) and hBMSC(Luc) cell groups, respectively).

Immunostaining of human $\beta$-2-microglobulin enabled visualization of the implanted cells still present in sections of constructs explanted at 8 weeks (Figure 4c). Positive human cells of both cell groups were detected as bundles at different locations in the tissue surrounding the coral granules. In addition, some human cells were observed along the periphery of the coral particles and embedded in osteocyte lacunae (arrows; Figure 4c). In fact, the presence of labelled cells was found in cell constructs apart from the presence of bone tissue. However, when bone tissue was present, it was consistently close and/or surrounded by labelled cells confirming the relationship between implanted cells and new bone tissue formation.

3.4 The secretome of SAD+ cells is both proangiogenic and chemoattractant but their potential does not exceed that of the hBMSCs

The proangiogenic potential of the secretome of both cell groups tested was evaluated using HUVEC cultured on Matrigel@, in the presence of conditioned media derived from either SAD+ cells or hBMSCs exposed to near-anoxia $(0.1 \% 02)$ for 7 days. Examination of the Matrigels ${ }^{\circledR}$ under light microscopy showed that the serum-containing medium (positive control), but not the serum-free medium (negative control), induced formation of a dense network of capillarylike tubes (Figure $5 \mathrm{aA}$ and $5 \mathrm{aB}$ ). Both conditioned media from sAD+ and hBMSC cells induced formation of vascular-like structures, but at a lesser extent than the formation observed when the positive control medium was used (Figure 5ac and $\mathrm{aD}$ ). Quantification proved that the numbers of vascular-like tubes per well induced by both condition ed media were similar (Figure 5b). In addition, the numbers of blood vessels quantified on histological sections from both sAD+ cell and hBMSC constructs ectopically implanted in mice for 8 weeks (Figure 5c) were also similar (Figure 5d). 
Moya et al.

The chemoattractant potential of the conditioned media derived from the two cell groups tested on fresh hBMSCs was evaluated using the migration test in Boyden chambers. Quantification of the number of migrated hBMSCs showed that the conditioned media from both the SAD+ and hBMSC cells induced migration of fresh hBMSCs as efficiently as the serumcontaining medium; the cell migration results induced by the two supernatants media tested were similar (Figures $5 \mathrm{e}-\mathrm{f}$ ).

\section{5 hBMSCs indirectly co-cultured with SAD+ cells exhibit higher osteogenic potential in vitro}

The potential of SAD+ cells to promote the osteogenic differentiation of recruited osteoprogenitors through paracrine effects was also investigated. To this aim, hBMSCs were co-cultured (without cell-cell contact) with either sAD+ or hBMSC cells in osteogenic media using a diffusion chamber (not permitting cell migration) for up to 21 days. The gene expression of the co-cultured hBMSCs was evaluated at Day 7 and expressed as a fold increase compared with results obtained using undifferentiated hBMSCs (at Day 0). The mRNA levels of the ALP and IBSP genes were enhanced in the hBMSCs co-cultured with either SAD+ cells or hBMSCs, whereas RUNX2 was unchanged and BGLAP decreased (Figure 6a). The foldchange of IBSP was significantly higher in the hBMSCs cocultured with sAD+ cells than with hBMSCs (82-fold vs 30 -fold induction, respectively); the fold-changes of the other genes tested were similar in the two groups tested (Figure 6a). Of note, only the fold-change in the expression of the adipogenic related-gene LPL was significantly higher in the case of hBMSCs/sAD+ cells than with hBMSCs/hBMSCs (16- and 6-fold induction, respectively; data not shown).

The ALP activity in the hBMSCs co-cultured with SAD+ cells was significantly higher than in those co-cultured with hBMSCs (Figure 6b). In addition, although no matrix mineralization was observed in hBMSCs co-cultured with hBMSCs for 21 days (Figure 6cA-D), a small amount of matrix mineralization was detected as early as Day 14 of osteogenic differentiation in hBMSCs co-cultured with sAD+ cells (Figure 6cE-F); mineralization in this co-culture increased after 21 days (Figure $6 \mathrm{cG}-\mathrm{H}$ ). Absorbance values of the eluted stain indicated a 3 -fold greater value in hBMSCs/sAD+ cells than in hBSMCs/hBMSCs after 21 days of culture (Figure $6 \mathrm{~d}$ ).

\section{DISCUSSION:}

In the present study, we evaluated the benefits of an adipogenic predifferentiation of hBMSCs on their proosteogenic potential both in vitro and in vivo. Our results provided evidence that, compared with unprimed hBMSCs, select adipogenic preconditioned hBMSCs (sAD+ cells) exhibited enhanced bone formation capability in an ectopic mouse model. This result may be explained by the commitment of such a predifferentiated SAD+ cell population to both the osteogenic and adipogenic lineages as well as by their proosteogenic paracrine effect on undifferentiated hBMSCs.

The current standard method to assess the bone-forming potential of a cell population in vivo is to load these cells onto ceramics and implant the cell constructs subcutaneously into immunocompromised mice (Goshima, Goldberg, \& Caplan, 1991). For this purpose, in the present study, trypsinization and reseeding of the hBMSC-Ad+ cells after the 14-day adipogenic differentiation period were necessary steps to prepare the cell-containing constructs. The outcome from this procedure was a select, but still heterogenous, adherent sAD+ cell population, whose phenotypic characterization provided evidence that they are between undifferentiated hBMSCs and unselected adipogenic differentiated hBMSCs (hBMSC-Ad+). The sAD+ cells (a) displayed spindle-shape morphology and contained a few lipid vesicles, (b) expressed CD73 and CD105 surface antigens at a lesser extent than the undifferentiated hBMSCs but at higher level than the hBMSC-Ad+ cells, and (c) expressed PPAR $\gamma$ and C/EBP $\alpha$ (two key transcriptome factors regulating the adipogenic differentiation) as well as other adipogenic-related genes (specifically, leptin and LPL), albeit, at a much lesser extent than the hBMSC-Ad+ cells. These data suggested that the most adipogenic-committed cells were eliminated during the cell trypsinization, most likely because of their weaker adherence onto substrate surfaces. It is possible that elimination of the most mature adipocytes from the selected adipogenic differentiated sAD+ cells might have an impact on their osteogenic potential. Indeed, mature adipocytes were reported to have a detrimental effect on boneforming cells (Rosen \& Bouxsein, 2006) and, subsequently, on bone formation in vivo.

An interesting result of the present study was that the sAD+ cells exhibited several hallmarks of osteogenic differentiation. These cells exhibited upregulated expression of the ALP and IBSP genes, the encoded proteins of which are both involved at an early stages of the mineralization process (Baht, Hunter, \& Goldberg, 2008; Golub \& Boesze-Battaglia, 2007); however, they did not upregulate bGLAP (osteocalcin), which is a marker of late osteoblast differentiation (Aubin, 2001). When cultured in osteogenic medium, sAD+ cells differentiated along the osteogenic lineage much faster than undifferentiated hBMSCs; these cells exhibited increased ALP activity and matrix mineralization detectable 2 weeks earlier than that expressed by the hBMSCs. Taken together, these results indicate that the select adipogenic-predifferentiated SAD+ cells were committed towards both the osteogenic and the adipogenic lineages. Analysis of the osteogenic-related gene expression and subsequent comparison with the select osteogenic differentiated sOS+ cells suggest that the SAD+ cells displayed a pre-osteoblastic phenotype slightly less mature than that expressed by the sOS+ cells.

Several literature reports have demonstrated that the adipogenic and osteogenic differentiation commitments of BMSCs are finely balanced and competitive. Fat-induction factors inhibit osteogenesis, and, conversely, bone-induction factors hinder adipogenesis, thereby, maintaining the undifferentiated state of BMSCs (Chen et al., 2016; Muruganandan et al., 2009). Specifically, RUNX2, one of the main determinant transcription factors for BMSC osteoblastogenesis, silences adipogenesis; knockout RUNX2 expression was reported to enhance adipogenesis (Jeon et al., 2003). Likewise, PPAR $\gamma$, the master regulator of adipogenesis, was reported to downregulate RUNX2 expression and to bind to the RUNX2 protein to inhibit transactivation of the osteogenic promoters (Rosen \& MacDougald, 2006). However, the adipogenic and osteogenic pathways also share common activators. For example, the BMP signalling pathway has a dual 
Moya et al.

role in regulating both the adipogenic and osteogenic differentiation of stem cells (Tang, Otto, \& Lane, 2004; zur Nieden, Kempka, Ran court, \& Ahr, 2005). Moreover, a number of in vitro studies showed a marker overlap between BMSCderived adipocytes and osteoblasts (Kollmer et al., 2013; Schilling et al., 2007) even at a single cell level (Ponce et al., 2008). For instance, it was reported that the ALP activity present within human preadipocytes increases with adipogenesis (Ali et al., 2006; Bianco, Costantini, Dearden, \& Bonucci, 1988). In the present study, single-cell analyses on sAD+ cells using flow cytometry provided evidence that $45 \%$ of lipid-containing SAD+ cells express the ALP protein (compared with $57 \%$ for lipid-free sAD+), confirming the link between ALP and adipogenesis. However, further analyses are needed to ensure that these lipid-containing and lipid-free cell populations both display a pre-osteoblastic phenotype.

The most interesting result of the present study is the enhanced bone-forming ability exhibited by the sAD+ cells in vivo. Subcutaneous implantation of the sAD+ cells (combined with coral particles) into nude mice resulted in strong increased de novo bone formation (11/11 vs. 6/14) and to a 5fold increase in the amount of deposited bone tissue in SAD+ cell-constructs compared with pertinent results obtained from undifferentiated hBMSCs. Furthermore, such beneficial effect of the adipogenic predifferentiation on hBMSCs was proved to be donor independent, as SAD+ cell-constructs prepared with cells from three individual donors all exhibited higher bone amounts than those obtained with undifferentiated hBMSCs.

The underlying mechanisms responsible for the beneficial effects of sAD+ cells on new bone formation in vivo were also investigated. Because a $(\sim 10 \%)$ portion of sAD+ cells contains lipids, we first investigated whether such lipid storage (by providing an extra energy source to the sAD+ cells) could help these cells to sustain their viability during the ischaemic episode which occurs after implantation. The results showed similar survival rates of the SAD+ cells and of the hBMSCs. In vitro, all cells from both groups died over the 14 days of culture, although in vivo, the number of viable implanted hBMSC(Luc) and sAD+(Luc) cells decreased over time to reach a survival rate of $15 \%-25 \%$ after 30 days of implantation. The latter finding is in agreement with published studies which have reported large loss of BMSCs post-implantation (Giannoni et al., 2009; Manassero et al., 2016). For this reason, the enhanced ectopic bone formation induced by the sAD+ cells, observed in this study, was not related to improved cell survival post-implantation.

In stem cell-based tissue engineering, hBMSCs play not only a direct role on new tissue formation owing to their ability to differentiate into functional tissue cells but also an indirect role by supporting resident progenitor cells via paracrine mechanisms (including the release of multiple immunomodulatory, angiogenic, chemotactic, and differentiating factors) (Baraniak \& McDevitt, 2010). In the present study, we hypothesized that the beneficial effect of sAD+ cells on new bone formation was due to both their higher osteogenic potential and pro-osteogenic paracrine effect on host hBMSCs.

First, taking advantage of their "already committed" state along the osteogenic lineage (in addition to their adipogenic committed state), the sAD+ cells may directly contribute to the process of new bone formation. Despite the observed weak survival rate of implanted cells in vivo, the presence of human cells in the vicinity of the newly formed bone tissue as well as in some osteocyte lacunae proved, indeed, their direct involvement in bone formation.

Second, the SAD+ cells likely contribute to the bone formation indirectly through the action of pro-osteogenic paracrine mediators onto host progenitors. This explanation is further supported by the fact that adipocytes are highly secretory cells. Indeed, in vitro studies demonstrated that adipocyte-secreted mediators, including hormones (such as leptin and adiponectin) and fatty acids, may affect the proliferation, function, and apoptosis of neighbouring cells (Elbaz, Rivas, \& Duque, 2009; Maurin et al., 2000).The functionality of the sAD+ secretome, especially its proangiogenic and chemoattractive potentials (two crucial paracrine properties necessary for the success of new bone tissue formation) was also assessed. The chemoattractive effects on hBMSCs as well as the proangiogenic effects on endothelial cells were similar for both the $\mathrm{SAD}+$ cell secretome and the hBMSC secretome. In addition, the number of invading blood vessels present in explanted cell constructs containing either the SAD+ or the hBMSC cells was similar. Based on this evidence, one may conclude that the bone forming potency of the sAD+ cells results neither from enhanced recruitment of host osteoprogenitors nor from enhanced vascularization of the implants.

The paracrine effects of sAD+ cells were further investigated to determine whether the sAD+ cells promoted the osteogenic differentiation of host progenitors. Co-culture experiments (to reproduce the indirect cell-to-cell interactions occurring in the cell constructs in vivo) revealed that, compared with the undifferentiated hBMSC secretome, the SAD+ paracrine factors upregulated the osteogenic-related IBSP gene expression of cocultured hBMSCs. Most importantly, both the ALP activity and matrix mineralization of these co-cultured hBMSCs were enhanced by the presence of $\mathrm{SAD}+$ cells. These results demonstrated the pro-osteogenic paracrine effect of the SAD+ cells on recruited host hBMSCs.

The pertinent mediators involved in the pro-osteogenic paracrine action of the SAD+ cells were not identified in the present study. Several recent studies have reported that several critical signalling pathways, including transforming growth factor-beta (TGF $\beta$ )/BMP, wingless-type MMTV integration site (Wnt), Hedgehogs (Hh), Notch, and fibroblast growth factors (FGFs) signalling pathways are involved in regulating both the adipogenic and osteogenic differentiation of MSCs. In addition to the well-known molecular signalling pathways, microRNAs were recently found to have roles in both osteogenic and adipogenic lineage commitment and terminal differentiation of hBMSCs (reviewed in Chen et al., 2016). In an attempt to identify the mediators involved in the sAD+ cells effect observed in the present study, expression of some key factors related to both the BMP and Wnt signalling pathways were compared between sAD+ cells and undifferentiated hBMSCs. Our data indicate that BMP-7 and BMP-9 gene were not expressed in any of the samples tested. Expression of BMP-2 and -6 was lower in SAD+ than in the hBMSC cells (Figure S6). Wnt1 and Wnt3a were not detected in any of the samples tested, whereas Wnt2 was less expressed in $\mathrm{sAD}+$ than in the hBMSC cells (Figure S6). Undoubtedly, further 
Moya et al.

research is needed in order to identify the crucial proosteogenic factors secreted by the sAD+ cells, which would be useful in optimizing stem cells bone tissue engineered constructs.

Altogether, our findings provided evidence that, compared with undifferentiated hBMSCs, select adipogenicpredifferentiated SAD+ cells expressed an enhanced osteogenic potency both in vitro and in vivo. In view of these observations, it would have been interesting to compare the in vivo osteogenic potency of sAD+ cells with that of osteogenic predifferentiated hBMSCs. Several research groups have already evaluated the potential benefit of the osteogenic induction of BMSCs prior to implantation on the bone formation in vivo; to date, however, the literature results are contradictory (Janicki et al., 2011; Kasten et al., 2005; Niemeyer et al., 2006). Direct comparison of the in vivo osteogenic performance of both adipogenic and osteogenic predifferentiated hBMSCs in cell constructs would enable elucidating the advantages of all these strategies.

\section{CONCLUSION}

Before BMSC-based bone tissue-engineering therapies can be widely used in the clinical arena, optimization of BMSCs in vitro preparation before transplantation must be addressed and determined. In the present study, we provided evidence that adipogenic-predifferentiated hBMSCs in tissue engineering constructs contribute to enhanced new bone tissue formation in vivo. Unexpectedly, such adipogenic priming endows hBMSCs with both high osteogenic potential and with pro-osteogenic paracrine-mediated function. Therefore, adipogenic preconditioning in ord er to improve the hBMSC osteogenic potency in vivo appears as a novel, efficient, and to date untapped, strategy for bone tissue engineering technology applications.

\section{ACKNOWLEDGMENTS}

The authors gratefully thank Professor R. Bizios for critically reading the manuscript. They also thank Françoise Pillier (UPR15-CNRS-LISE) for BSEM observations and the vectorology platform (INSERM US 005-CNRS UMS 3427-TBMCore) for the lentivirus production. They acknowledge the Centre National de la Recherche Scientifique (CNRS), the Direction Générale de l'Armement (DGA), and the Fondation pour l'Avenir for the financial support of the study

\section{DISCLOSURE STATEMENT}

The authors declare no competing financial interests

\section{REFERENCES}

Ali, A. T., Penny, C. B., Paiker, J. E., Psaras, G., Ikram, F., \& Crowther, N. J. (2006). The relationship between alkaline phosphatase activity and intracellular lipid accumulation in murine 3T3-L1 cells and human preadipocytes. Analytical Biochemistry, 354, 247- 254.

Aubin, J. E. (2001). Regulation of osteoblast formation and function. Reviews in Endocrine \& Metabolic Disorders, 2, 81- 94. Crossref CAS PubMed Google Scholar
Baht, G. S., Hunter, G. K., \& Goldberg, H. A. (2008). Bone sialoproteincollagen interaction promotes hydroxyapatite nucleation. Matrix Biology, 27, 600-608.

Baraniak, P. R., \& McDevitt, T. C. (2010). Stem cell paracrine actions and tissue regeneration. Regenerative Medicine, 5, 121- 143.

Becquart, P., Cambon-Binder, A., Monfoulet, L. E., Bourguignon, M., Vandamme, K., Bensidhoum, M., ... Logeart-Avramoglou, D. (2012). Ischemia is the prime but not the only cause of human multipotent stromal cell death in tissue-engineered constructs in vivo. Tissue Engineering. Part A, 18, 2084- 2094.

Bianco, P., Costantini, M., Dearden, L. C., \& Bonucci, E. (1988). Alkaline phosphatase positive precursors of adipocytes in the human bone marrow. British Journal of Haematology, 68, 401- 403.

Bruder, S. P., Jaiswal, N., Ricalton, N. S., Mosca, J. D., Kraus, K. H., \& Kadiyala, S. (1998). Mesenchymal stem cells in osteobiology and applied bone regeneration. Clinical Orthopaedics and Related Research, S247-S256.

Chen, Q., Shou, P., Zheng, C., Jiang, M., Cao, G., Yang, Q., ... Shi, Y. (2016). Fate decision of mesenchymal stem cells: Adipocytes or osteoblasts? Cell Death and Differentiation, 23, 1128-1139.

Dickhut, A., Gottwald, E., Steck, E., Heisel, C., \& Richter, W. (2008). Chondrogenesis of mesenchymal stem cells in gel-like biomaterials in vitro and in vivo. Frontiers in Bioscience, 13, 4517- 4528.

Elbaz, A., Rivas, D., \& Duque, G. (2009). Effect of estrogens on bone marrow adipogenesis and Sirt1 in aging C57BL/6J mice. Biogerontology, 10, 747- 755 .

Friedenstein, A. J., Chailakhjan, R. K., \& Lalykina, K. S. (1970). The development of fibroblast colonies in monolayer cultures of guinea-pig bone marrow and spleen cells. Cell and Tissue Kinetics, 3, 393- 403.

Ghali, O., Broux, O., Falgayrac, G., Haren, N., vanLeeuwen, J. P., Penel, G., ... Chauveau, C. (2015). Dexamethasone in osteogenic medium strongly induces adipocyte differentiation of mouse bone marrow stromal cells and increases osteoblast differentiation. BMC Cell Biology, 16, 9.

Giannoni, P., Scaglione, S., Daga, A., Ilengo, C., Cilli, M., \& Quarto, R. (2009). Short-time survival and engraftment of bone marrow stromal cells in an ectopic model of bone regeneration. Tissue Engineering. Part A, 16, 489- 499.

Golub, E. E., \& Boesze-Battaglia, K. (2007). The role of alkaline phosphatase in mineralization. Current Opinion in Orthopaedics, 18, 444-448.

Goshima, J., Goldberg, V. M., \& Caplan, A. I. (1991). Osteogenic potential of culture-expanded rat marrow cells as assayed in vivo with porous calcium phosphate ceramic. Biomaterials, 12, 253- 258.

Janicki, P., Kasten, P., Kleinschmidt, K., Egermann, M., Kasten, P., \& Richter, W. (2010). Chondrogenic pre-induction of human mesenchymal stem cells on beta-TCP: Enhanced bone quality by endochondral heterotopic bone formation. Acta Biomaterialia, 6, 3292- 3301

Janicki, P., Boeuf, S., Steck, E., Egermann, M., Kasten, P., \& Richter, W. (2011). Prediction of in vivo bone forming potency of bone marrowderived human mesenchymal stem cells. European Cells \& Materials, 21, 488- 507.

Jeon, M. J., Kim, J. A., Kwon, S. H., Kim, S. W., Park, K. S., Park, S. W., ... Shin, C. S. (2003). Activation of peroxisome proliferator-activated receptor-gamma inhibits the Runx2-mediated transcription of osteocalcin in osteoblasts. The Journal of Biological Chemistry, 278, $23270-23277$ 
Moya et al.

Jukes, J. M., Both, S. K., Leusink, A., Sterk, L. M., vanBlitterswijk, C. A., \& deBoer, J. (2008). Endochondral bone tissue engineering using embryonic stem cells. Proceedings of the National Academy of Sciences of the United States of America, 105, 6840-6845.

Kasten, P., Vogel, J., Luginbuhl, R., Niemeyer, P., Tonak, M., Lorenz, H., ... Richter, W. (2005). Ectopic bone formation associated with mesenchymal stem cells in a resorbable calcium deficient hydroxyapatite carrier. Biomaterials, 26, 5879- 5889.

Kollmer, M., Buhrman, J. S., Zhang, Y., \& Gemeinhart, R. A. (2013) Markers are shared between adipogenic and osteogenic differentiated mesenchymal stem cells. J Dev Biol Tissue Eng, 5, 18- 25.

Lee, Y. H., Chen, S. Y., Wiesner, R. J., \& Huang, Y. F. (2004). Simple flow cytometric method used to assess lipid accumulation in fat cells Journal of Lipid Research, 45, 1162-1167.

Logeart-Avramoglou, D., Oudina, K., Bourguignon, M., Delpierre, L., Nicola, M. A., Bensidhoum, M., ... Petite, H. (2010). In vitro and in vivo bioluminescent quantification of viable stem cells in engineered constructs. Tissue Engineering. Part C, Methods, 16, 447-458.

Manassero, M., Paquet, J., Deschepper, M., Viateau, V., Retortillo, J., Bensidhoum, M., ... Petite, H. (2016). Comparison of survival and osteogenic ability of human mesenchymal stem cells in orthotopic and ectopic sites in mice. Tissue Engineering. Part A, 22, 534- 544.

Maurin, A. C., Chavassieux, P. M., Frappart, L., Delmas, P. D., Serre, C. M. \& Meunier, P. J. (2000). Influence of mature adipocytes on osteoblast proliferation in human primary cocultures. Bone, 26, 485- 489.

Monfoulet, L. E., Becquart, P., Marchat, D., Vandamme, K., Bourguignon, M., Pacard, E., ... Logeart-Avramoglou, D. (2014). The pH in the microenvironment of human mesenchymal stem cells is a critical factor for optimal osteogenesis in tissue-engineered constructs. Tissue Engineering. Part A, 20, 1827-1840.

Muruganandan, S., Roman, A. A., \& Sinal, C. J. (2009). Adipocyte differentiation of bone marrow-derived mesenchymal stem cells: Cross talk with the osteoblastogenic program. Cellular and Molecular Life Sciences, 66, 236- 253.

zur Nieden, N. I., Kempka, G., Rancourt, D. E., \& Ahr, H. J. (2005) Induction of chondro-, osteo- and adipogenesis in embryonic stem cells by bone morphogenetic protein-2: Effect of cofactors on differentiating lineages. BMC Developmental Biology, 5, 1.

Niemeyer, P., Kasten, P., Simank, H. G., Fellenberg, J., Seckinger, A., Kreuz, P. C., ... Krause, U. (2006). Transplantation of mesenchymal stromal cells on mineralized collagen leads to ectopic matrix synthesis in vivo independently from prior in vitro

Pelttari, K., Winter, A., Steck, E., Goetzke, K., Hennig, T., Ochs, B. G., .. Richter, W. (2006). Premature induction of hypertrophy during in vitro chondrogenesis of human mesenchymal stem cells correlates with calcification and vascular invasion after ectopic transplantation in SCID mice. Arthritis and Rheumatism, 54, 3254- 3266.

Petite, H., Viateau, V., Bensaid, W., Meunier, A., dePollak, C. Bourguignon, M., ... Guillemin, G. (2000). Tissue-engineered bone regeneration. Nature Biotechnology, 18, 959- 963.

Pittenger, M. F., Mackay, A. M., Beck, S. C., Jaiswal, R. K., Douglas, R., Mosca, J. D., ... Marshak, D. R. (1999). Multilineage potential of adult human mesenchymal stem cells. Science, 284, 143-147.

Ponce, M. L., Koelling, S., Kluever, A., Heinemann, D. E., Miosge, N., Wulf, G., ... Siggelkow, H. (2008). Coexpression of osteogenic and adipogenic differentiation markers in selected subpopulations of primary human mesenchymal progenitor cells. Journal of Cellular Biochemistry, 104, $1342-1355$
Rosen, C. J., \& Bouxsein, M. L. (2006). Mechanisms of disease: Is osteoporosis the obesity of bone? Nature Clinical Practice. Rheumatology, 2, 35- 43 .

Rosen, E. D., \& MacDougald, O. A. (2006). Adipocyte differentiation from the inside out. Nature Reviews. Molecular Cell Biology, 7, 885896.

Schilling, T., Noth, U., Klein-Hitpass, L., Jakob, F., \& Schutze, N. (2007). Plasticity in adipogenesis and osteogenesis of human mesenchymal stem cells. Molecular and Cellular Endocrinology, 271, 1- 17.

Tang, Q. Q., Otto, T. C., \& Lane, M. D. (2004). Commitment of C3H10T1/2 pluripotent stem cells to the adipocyte lineage. Proceedings of the National Academy of Sciences of the United States of America, 101, 9607-9611.

Vaquette, C., Ivanovski, S., Hamlet, S. M., \& Hutmacher, D. W. (2013). Effect of culture conditions and calcium phosphate coating on ectopic bone formation. Biomaterials, 34, 5538- 5551.

Ye, X., Yin, X., Yang, D., Tan, J., \& Liu, G. (2012). Ectopic bone regeneration by human bone marrow mononucleated cells, undifferentiated and osteogenically differentiated bone marrow mesenchymal stem cells in beta-tricalcium phosphate scaffolds. Tissue Engineering. Part C, Methods, 18, 545- 556.

Zong, C., Xue, D., Yuan, W., Wang, W., Shen, D., Tong, X., ... Wang, J. (2010). Reconstruction of rat calvarial defects with human mesenchymal stem cells and osteoblast-like cells in poly-lactic-coglycolic acid scaffolds. European Cells \& Materials, 20, 109- 120.

\section{Corresponding author:}

Delphine Logeart-Avramoglou, Laboratory of Bioengineering and Bioimaging for Osteo-Articular tissues, UMR 7052 CNRS, Univ Paris Diderot, Sorbonne Paris Cité, Paris, France.

delphine.logeart@univ-paris-diderot.fr

\section{ORCID}

Adrien Moya http://orcid.org/0000-0002-3489-5215

Nathanaël Larochette http://orcid.org/0000-0002-7936-678X Marianne Bourguignon http://orcid.org/0000-0001-98241248

Esther Potier http://orcid.org/0000-0001-7919-2110

Hervé Petite http://orcid.org/0000-0003-1694-5060 Delphine Logeart-Avramoglou http://orcid.org/0000-00022052-

9425

How to cite this article: Moya A, Larochette N, Bourguignon $\mathrm{M}$, et al. Osteogenic potential of adipogenic predifferentiated human bone marrow-derived multipotent stromal cells for bone tissue-engineering. J Tissue Eng Regen Med. 2017;1-14. https://doi.org/10.1002/term.2571 


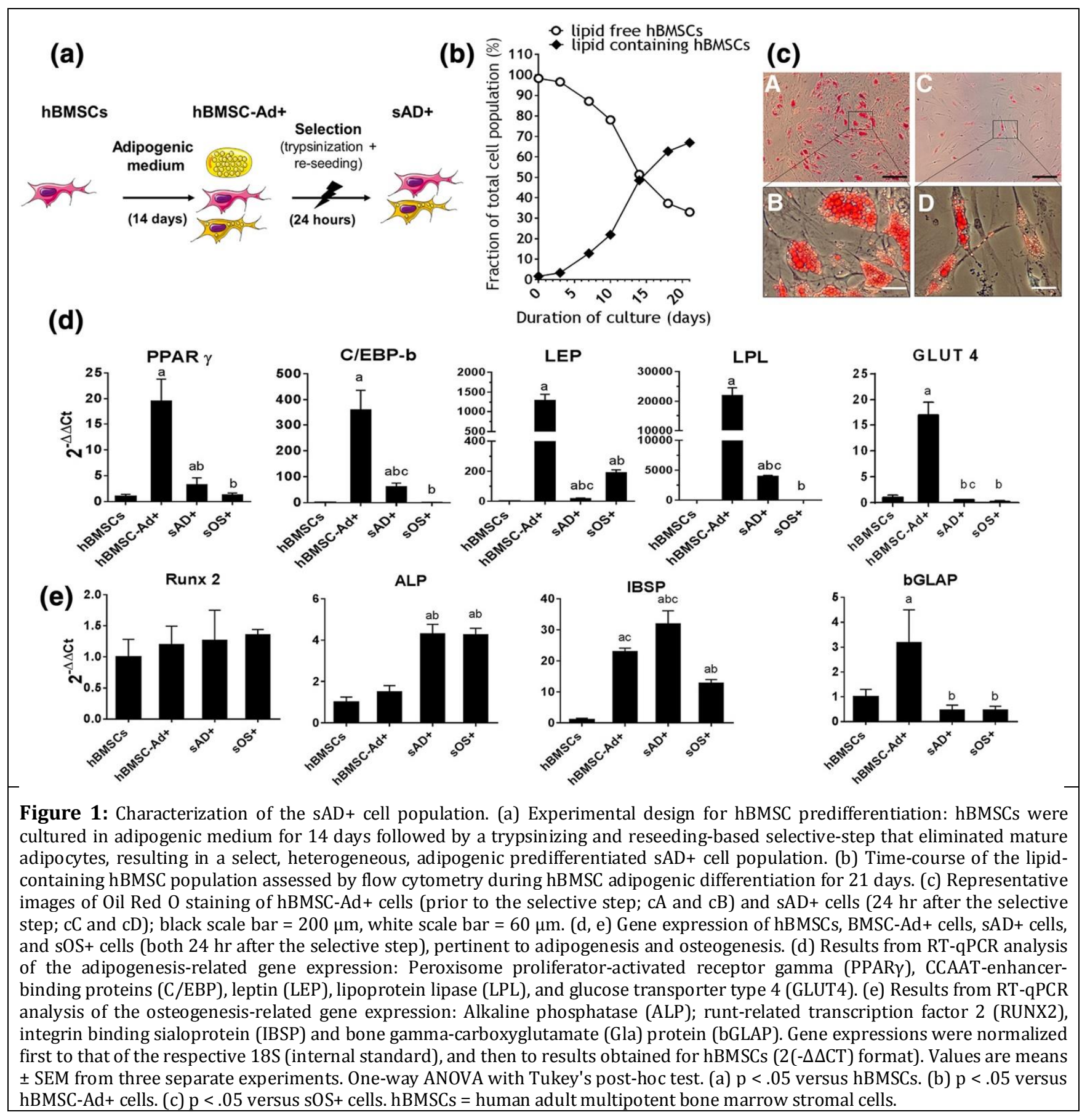




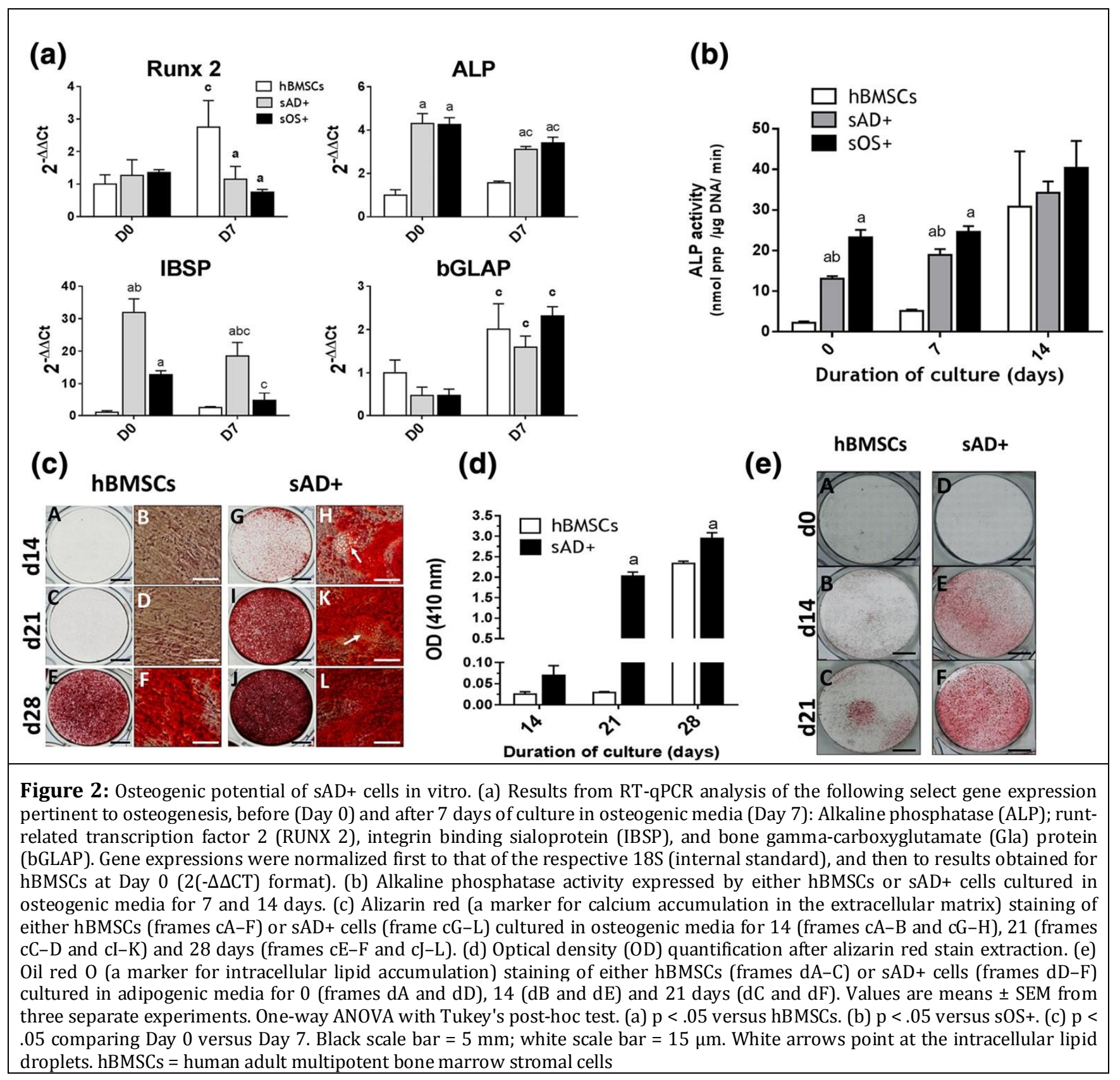


(a)
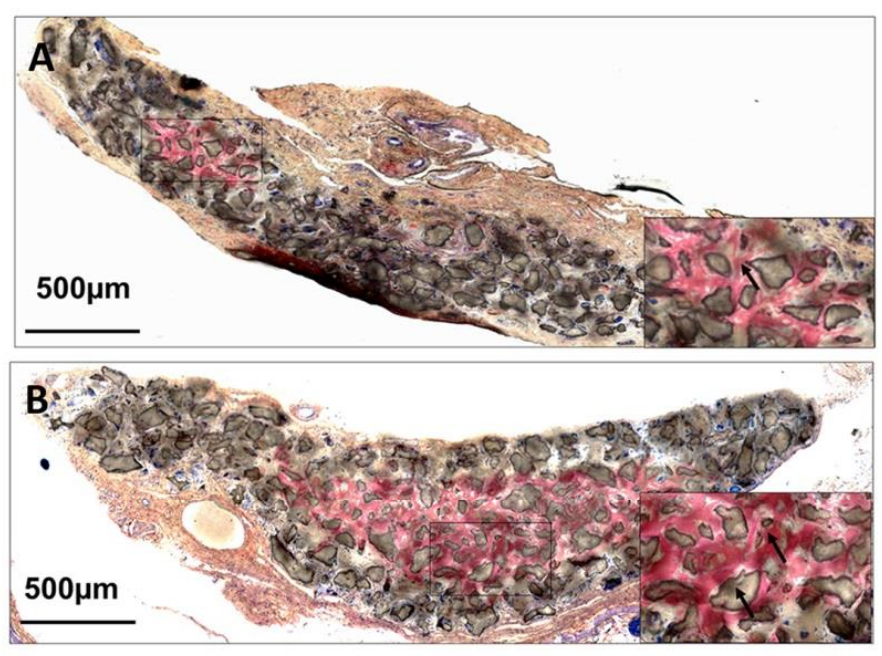

(b)

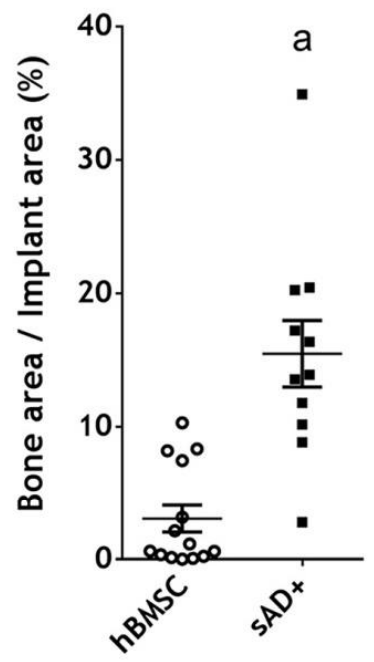

(c)

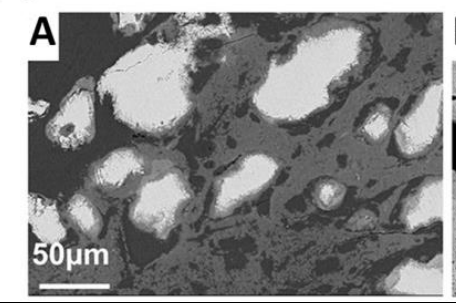

(d)

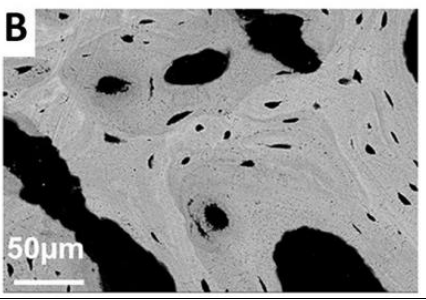

\begin{tabular}{|l|c|c|c|}
\hline \multicolumn{1}{|c|}{ Element } & Symbol & $\begin{array}{c}\text { Bone in sAD+ cell- } \\
\text { containing construct }\end{array}$ & $\begin{array}{c}\text { Murine } \\
\text { cortical bone }\end{array}$ \\
\hline Oxygen & $\mathrm{O}$ & $71.8 \%$ & $64.2 \%$ \\
Calcium & $\mathrm{Ca}$ & $16.1 \%$ & $21.3 \%$ \\
\hline Phosphorus & $\mathrm{P}$ & $11.4 \%$ & $13.2 \%$ \\
Sodium & $\mathrm{Na}$ & $0.7 \%$ & $0.8 \%$ \\
Magnesium & $\mathrm{Mg}$ & $0.0 \%$ & $0.5 \%$ \\
\hline Total & & $100 \%$ & $100 \%$ \\
\hline
\end{tabular}

Figure 3: De novo bone formation in tissue engineered constructs containing either hBMSCs or sAD+ cells. The constructs were seeded with either hBMSCs or sAD+ cells on coral particles and implanted ectopically in nude mice for 8 weeks. Representative histology sections of constructs containing either hBMSCs (aA) or sAD+ cells (aB) stained with Picrofuchsin and Stevenel's blue. (b) Quantification of the bone area as a percentage of the total implant area. Values are means \pm SEM $(n=11$ for sAD+ cells; $n=14$ for hBMSCs). Mann-Whitney test; (a) $p<.001$ comparing sAD+ cells versus hBMSCs. (c) SEM back-scattered images of sAD+ cellcontaining construct (cA) in comparison with a nude mouse cortical bone (cB). The light regions on the image indicate higher mineralization. (d) Chemical composition of bone tissue in the sections. The corresponding Ca/P ratio in bone tissue from sAD+ cell-containing construct and mouse cortical bone was 1.41 and 1.61, respectively, indicating the immature aspect of the newly formed bone tissue in $\mathrm{SAD}+$ construct 
Moya et al.

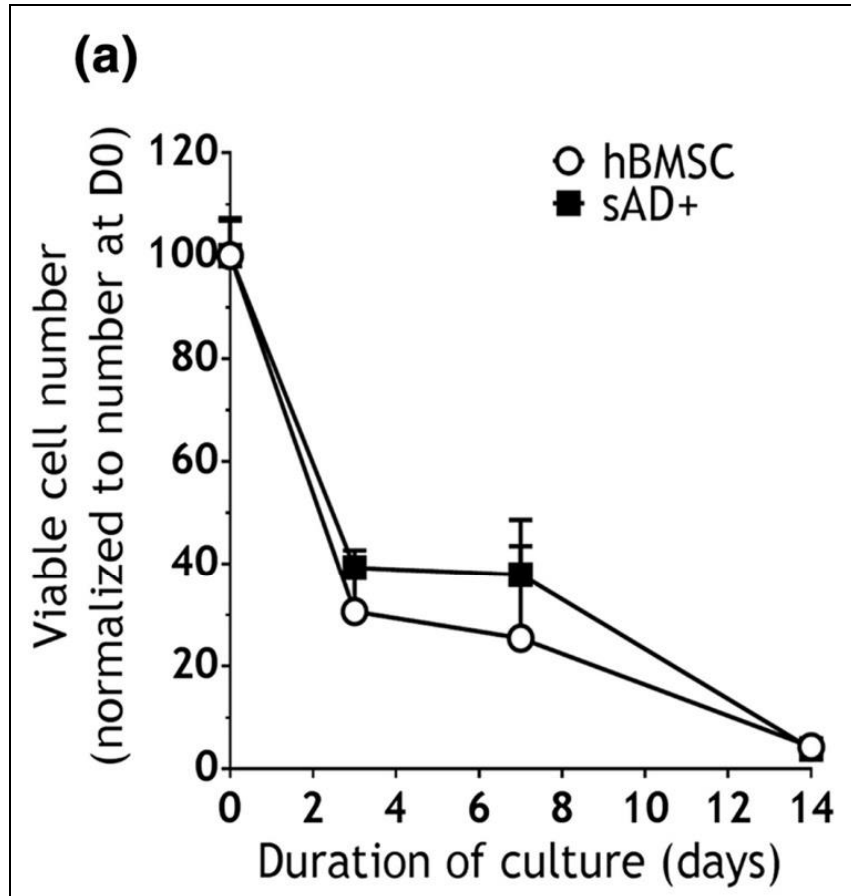

(c)

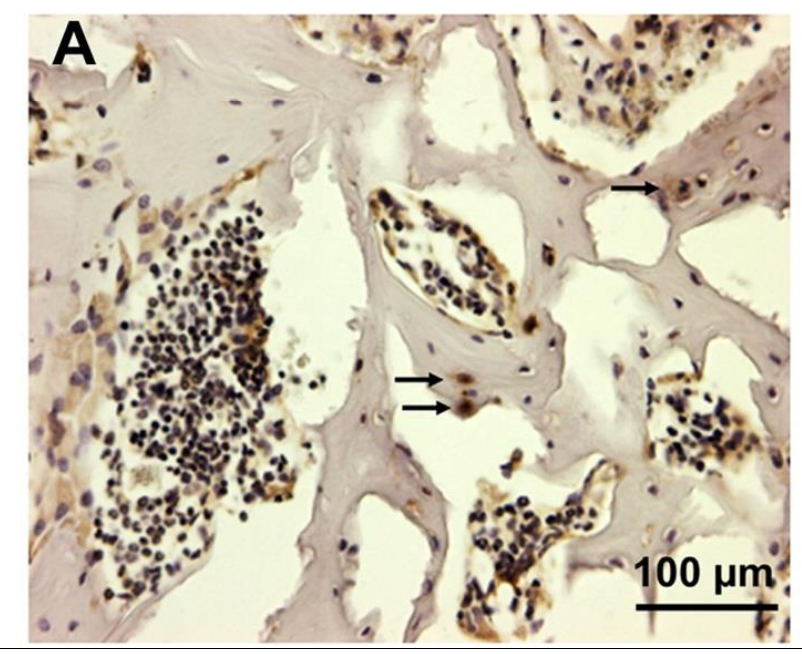

(b)

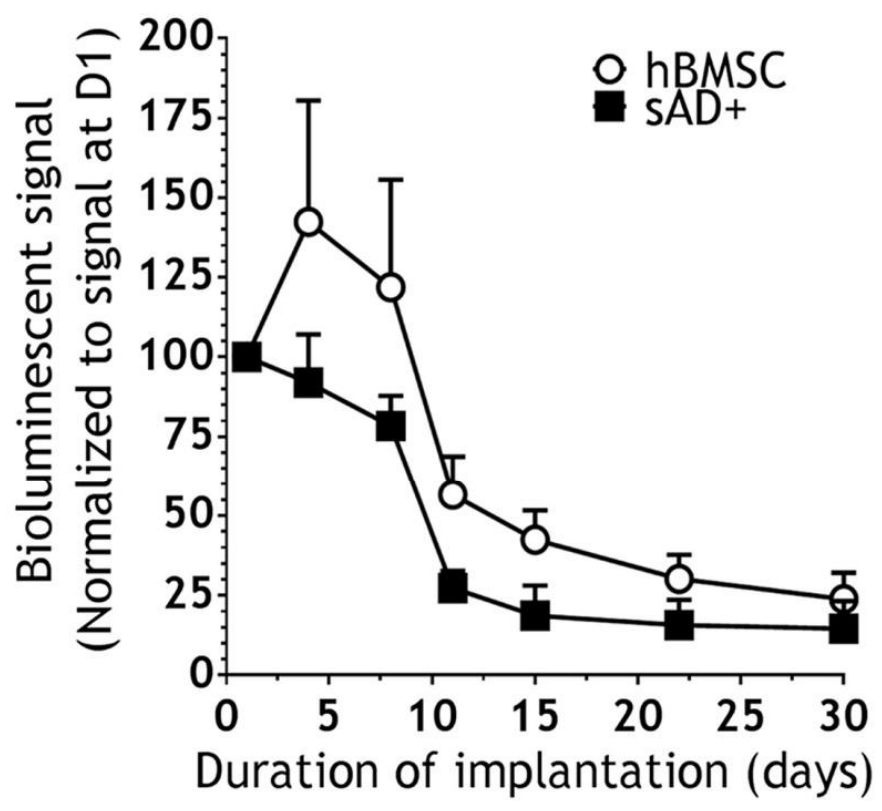

SAD+

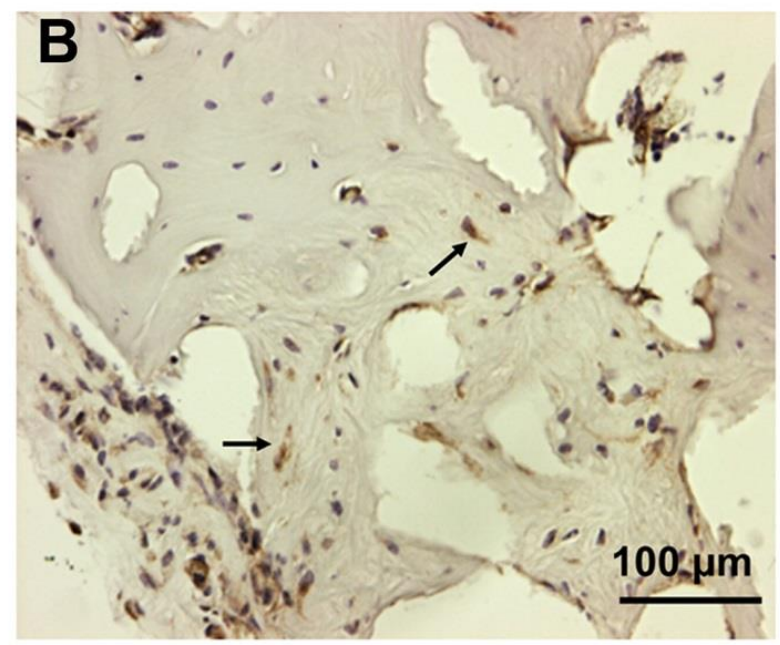

Figure 4: Fate of the SAD+ and hBMSC cells under ischaemic conditions in vitro and in vivo. (a) Time-course of the cell viability of both hBMSCs and SAD+ cells cultured under (near-anoxia, glucose depletion, and serum depletion) ischaemic conditions. The cell viability was assessed after Hoescht 33442-staining (total nuclei) and propidium iodide-staining (dead cells) followed by flow cytometry cells analysis. The values are means \pm SEM. $(n=6)$. Two-ways ANOVA, $p>.05$. (b) In vivo cell viability of both hBMSCs(Luc) and sAD+(Luc) cells determined by bioluminescent imaging after ectopic implantation in nude mice for 30 days. The photon fluxes were normalized to those obtained at Day 1 post-implantation. The values are means \pm SEM. (n $=6)$. Two-way ANOVA, $p>.05$ (c) Implanted hBMSC (frame cA) or SAD+ cells (frame cB), still present in the constructs explanted 8 weeks postimplantation were detected on paraffin-treated sections following immunostaining against human $\beta$-2-microglobulin. The black arrows points at positive labelled osteocytes. hBMSCs = human adult multipotent bone marrow stromal cells 
(a)

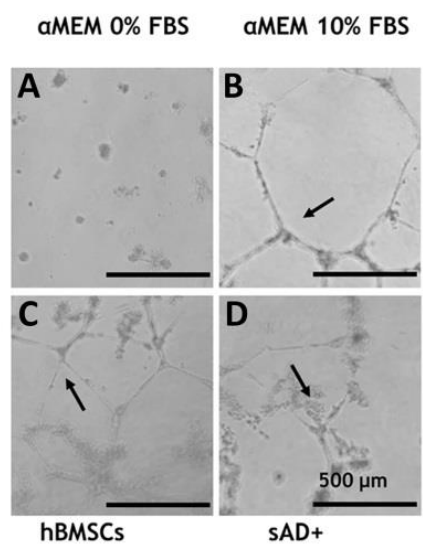

(c)
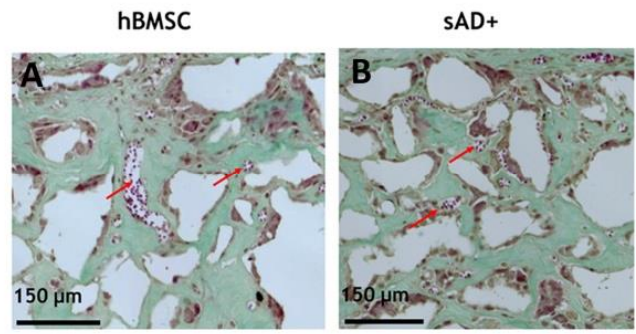

(b)

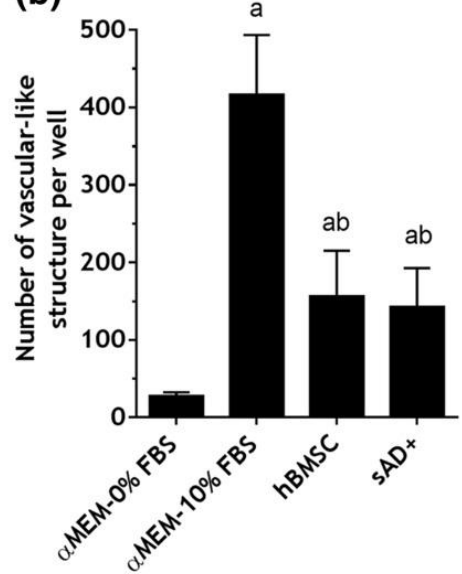

(d)

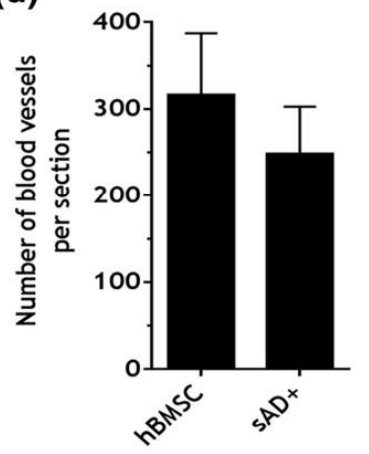

(e)
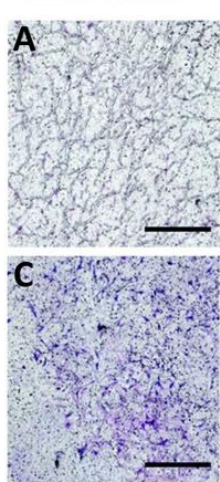

hBMSC
aMEM- $10 \%$ FBS

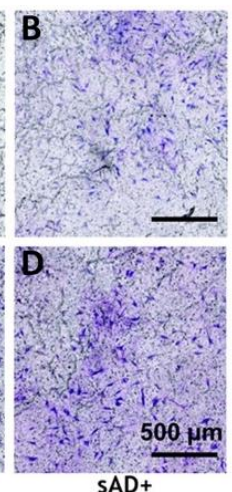

(f)

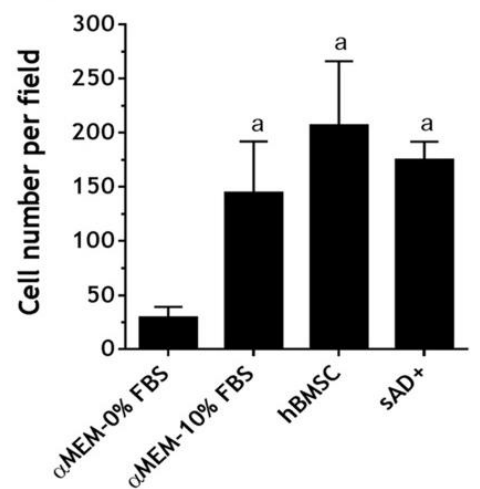

Figure 5: Angiogenic and chemo-attractive potentials of the sAD+ cells. (a) Representative light micrographs illustrating formation and (b) quantification of vascular-like structures (black arrows in a) formed by human umbilical vein endothelial cells on Matrigel matrices in the presence of $\alpha \mathrm{MEM}-0 \% \mathrm{FBS}$ (frame aA; negative control), $\alpha \mathrm{MEM}-10 \% \mathrm{FBS}$ (frame aB; positive control), supernatant conditioned media obtain ed from either hBMSCs (frame aC) or sAD+ cells (frame aD) cultured with glucose (5 g/L) in a $0.1 \%$ oxygen environment for 7 days. Values are means \pm SEM from two separate experiments $(\mathrm{n}=6$ total). One-way ANOVA with Tukey's post-hoc test. (a) $\mathrm{p}<.05$ conditioned media versus $\alpha$ MEM-0\%FBS (b) $\mathrm{p}<.05$ conditioned media vs. $\alpha$ MEM-10\%FBS. (c) Representative histology sections of constructs containing either hBMSC (frame cA) or SAD+ (frame cB) cells stained with Masson's trichrome. Red arrows point at a blood vessel. (d) Quantification of the number of blood vessels determined throughout the section. Values are means \pm SEM ( $n=10$ per implant). Mann-Whitney test: $p>.05$. (e) Representative light micrographs and (f) quantification of migrated hBMSCs across the porous membrane in Boyden chambers. The bottom well was filled with either $\alpha \mathrm{MEM}-0 \%$ FBS (frame eA; negative control), $\alpha \mathrm{MEM}-10 \% \mathrm{FBS}$ (frame eB; positive control), or supernatant conditioned media obtained from either hBMSCs (frame eC) or sAD+ cells (frame eD). Values are means \pm SEM from two separate experiments $(n=6$ total). One-way ANOVA with Tukey's post-hoc test: (a) $\mathrm{p}<.05$ conditioned media vs. $\alpha$ MEM-0\%FBS 
Moya et al.
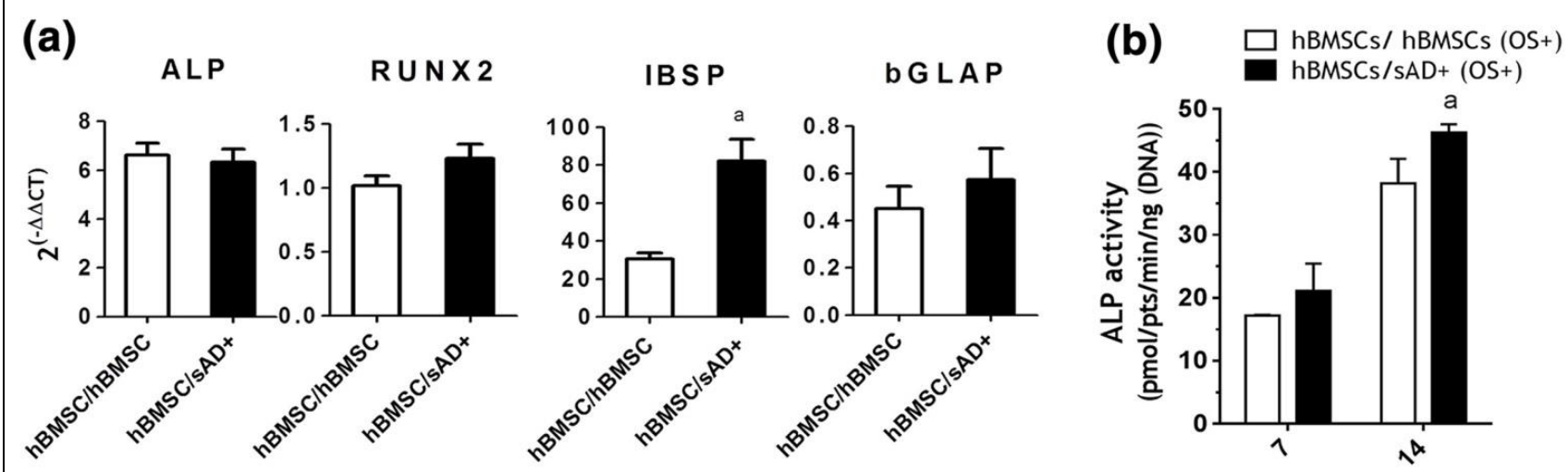

Duration of culture (days)

(c) hBMSC /hBMSCs (OS+)

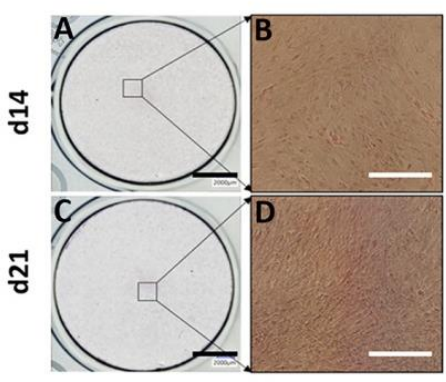

hBMCs $/ \mathrm{sAD}+(\mathrm{OS}+)$

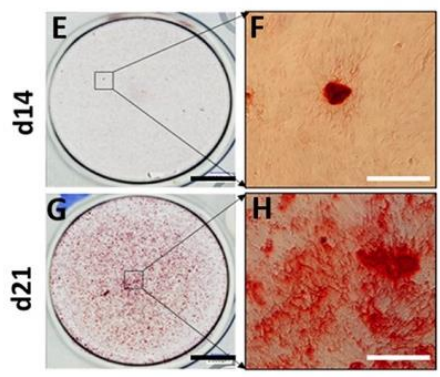

hBMSCs / hBMSCs (OS+) $\mathrm{hBMSCs} / \mathrm{sAD}+(\mathrm{OS}+)$

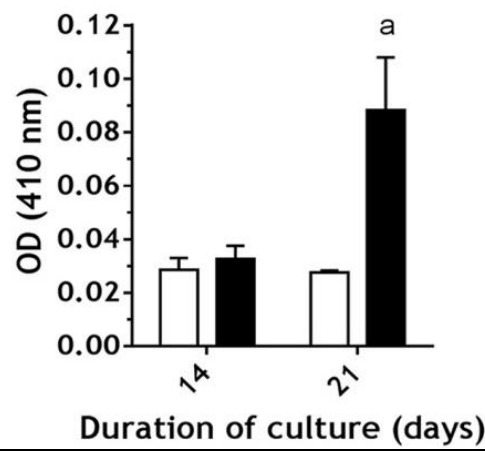

Figure 6: Osteogenic potential of hBMSCs indirectly co-cultured with sAD+ cells. (a) Results from RT-qPCR analysis of select gene expression pertinent to osteogenesis after 7 days of culture in osteogenic media for hBMSCs co-cultured with either hBMSCs (hBMSC/hBMSC) or SAD+ cells (hBMSC/sAD+): Alkaline phosphatase (ALP); runt-related transcription factor 2 (RUNX2), integrin binding sialoprotein (IBSP), and bone gamma-carboxyglutamate (Gla) protein (bGLAP). The gene expression was normalized first to that of the respective $18 \mathrm{~S}$ (internal standard), and then to results obtained with undifferentiated hBMSCs at Day 0 (2(- $\Delta \Delta \mathrm{CT}$ ) format). Values are means \pm SEM from three separate experiments $(n=6$ total). Mann-Whitney test: (a) $p<.05$ versus hBMSC/hBMSC. (b) Alkaline phosphatase activity in hBMSCs co-cultured with either hBMSCs or sAD+ cells in osteogenic media for 7 and 14 days. Values are means \pm SEM $(n=3)$. Mann-Whitney test: (a) $p<.05$ versus hBMSC/hBMSC. (c) Alizarin red staining of hBMSCs co-cultured with either hBMSC (frames cA-D) or sAD+ cells (frames cE-H) and in osteogenic media for 14 (frames cA-B and $\mathrm{cE}-\mathrm{F}$ ) and 21 days (frames $\mathrm{cC}-\mathrm{D}$ and $\mathrm{cG}-\mathrm{H}$ ). (d) Optical density (OD) quantification after alizarin red stain extraction. Values are means \pm SEM $(\mathrm{n}=3)$. Mann-Whitney test, $(\mathrm{a}) \mathrm{p}<.05$ versus hBMSC/hBMSC 


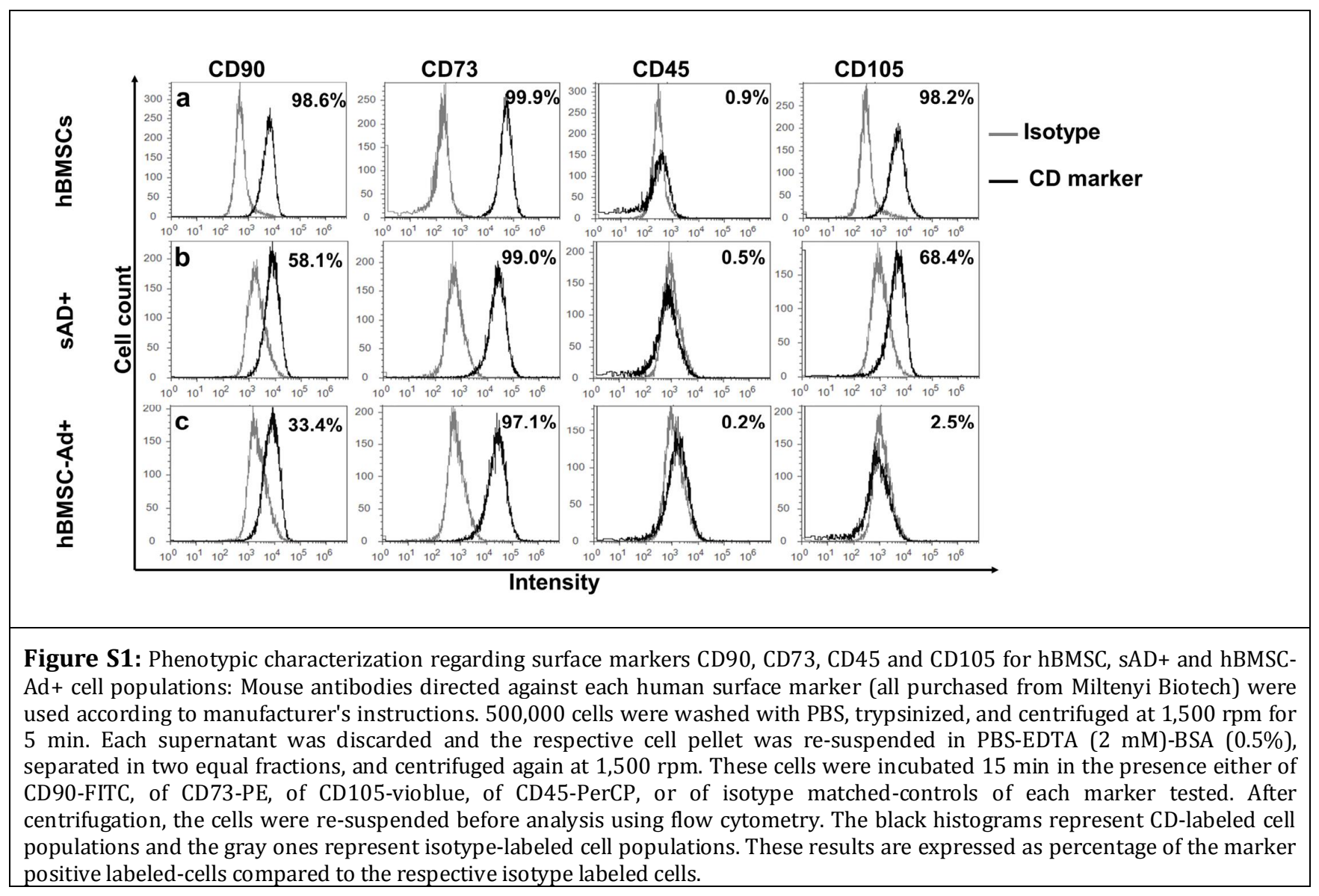

A

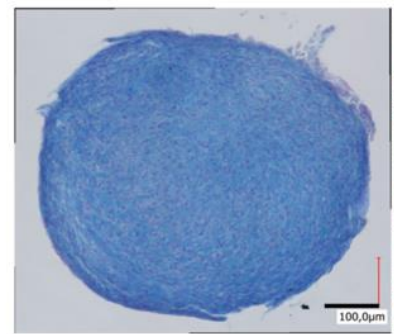

Chondrogenic differentiation
B

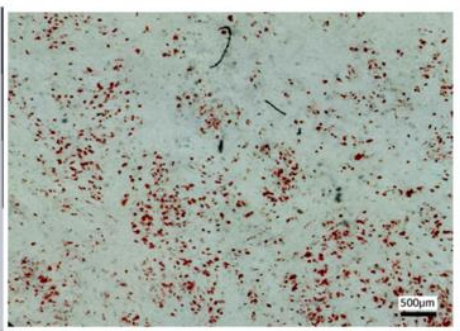

Adipogenic differentiation
C

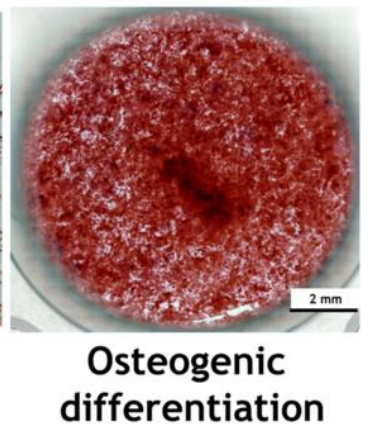

Figure S2: Multilineage potential of hBMSCs. (A): For the chondrogenic differentiation 2,5x104 hBMSC cells were cultured in micromass pellets in the presence of high-glucose DMEM medium containing $50 \mu \mathrm{g} / \mathrm{ml}$ ascorbic acid-2 phosphate, $100 \mathrm{nM}$ dexamethasone, $1 \%(\mathrm{v} / \mathrm{v}$ ) ITS and $10 \mathrm{ng} / \mathrm{ml}$ TGF 33 for 21 days (Mackay et al. Tissue eng, 1998). At that time, the hBMSCs cell aggregates were fixed, embedded in paraffin and processed for histochemical analysis; tissue section was stained with Alcian Blue, to detect sulfated proteoglycans (scale $100 \mu \mathrm{m}$ ). (B) For adipogenic and (C) osteogenic differentiation potentials, hMSCs were cultured either in an adipogenic (AD) medium for 21 days and stained with oil red 0 solution (scale $500 \mu \mathrm{m}$ ) or in an osteogenic (OS) medium for 28 days and stained with alizarin red (scale $2000 \mu \mathrm{m}$ ) as described in the Materials and Methods section. 

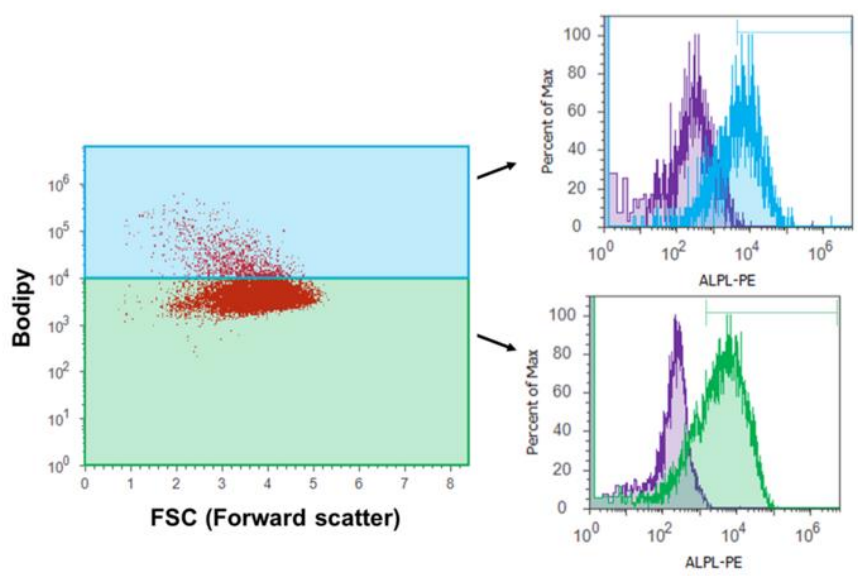

\begin{tabular}{|c|c|c|}
\hline \multirow{2}{*}{ hBMSC } & \multicolumn{2}{|c|}{$\begin{array}{c}\text { ALP+ cells within } \\
\text { each population } \\
\text { Bodipy- }\end{array}$} \\
\cline { 2 - 3 } Bodipy+ \\
hBMSC-Ad+ & $16 \%$ & NA \\
SAD+ & $47 \%$ & $27 \%$ \\
SOS+ & $57 \%$ & $45 \%$ \\
\hline
\end{tabular}

NA : not applicable (Bodipy (-) cells)

Purple : PE antibody isotype Blue/green : ALPL-PE antibody

Figure S3: Phenotypic characterization regarding both lipid content and ALP labeling for hBMSC-derived cell populations 200,000 of either hBMSCs, hBMSC-Ad+, sAD+, or sOS+ cells were washed with PBS, trypsinized, and centrifuged at 1,500 rpm for 5 min. Each supernatant was discarded and the respective pellet cells were incubated with $0.5 \mu \mathrm{g} / \mathrm{ml}$ Bodipy $493 / 503$ (a marker of lipid droplets, Thermo Fisher Scientific) together either with $10 \mu \mathrm{l}$ of anti-human phycoerythrin (PE)-conjugated ALPL antibody (\# FAB1448P, Biotechne) or with its isotype matched-control for 15 min at $4^{\circ} \mathrm{C}$. (A) For hBMSC-Ad+ and sAD+ cell populations, ALP(+) cells were monitored on both Bodipy ( + ) and Bodipy(-) cell sub-populations (gate adjusted in the FSC (Forward Scatter) versus Blue laser 1 (BL1) channel plot) using an Attune cytometer (Applied Biosystems). The blue and green histograms represent Bodipy(+)/ALPL(+) and Bodipy(-)/ALPL(+) cell populations, respectively, and the purple ones represent isotype-labeled cell populations. Since hBMSCs and sOS+ cell populations did not contain lipid droplets, analysis of ALP(+) cells was performed only on Bodipy(-) cells. (B) The results are expressed as percentage of the ALPL positive labeled-cells compared to the respective isotype labeled cells. 


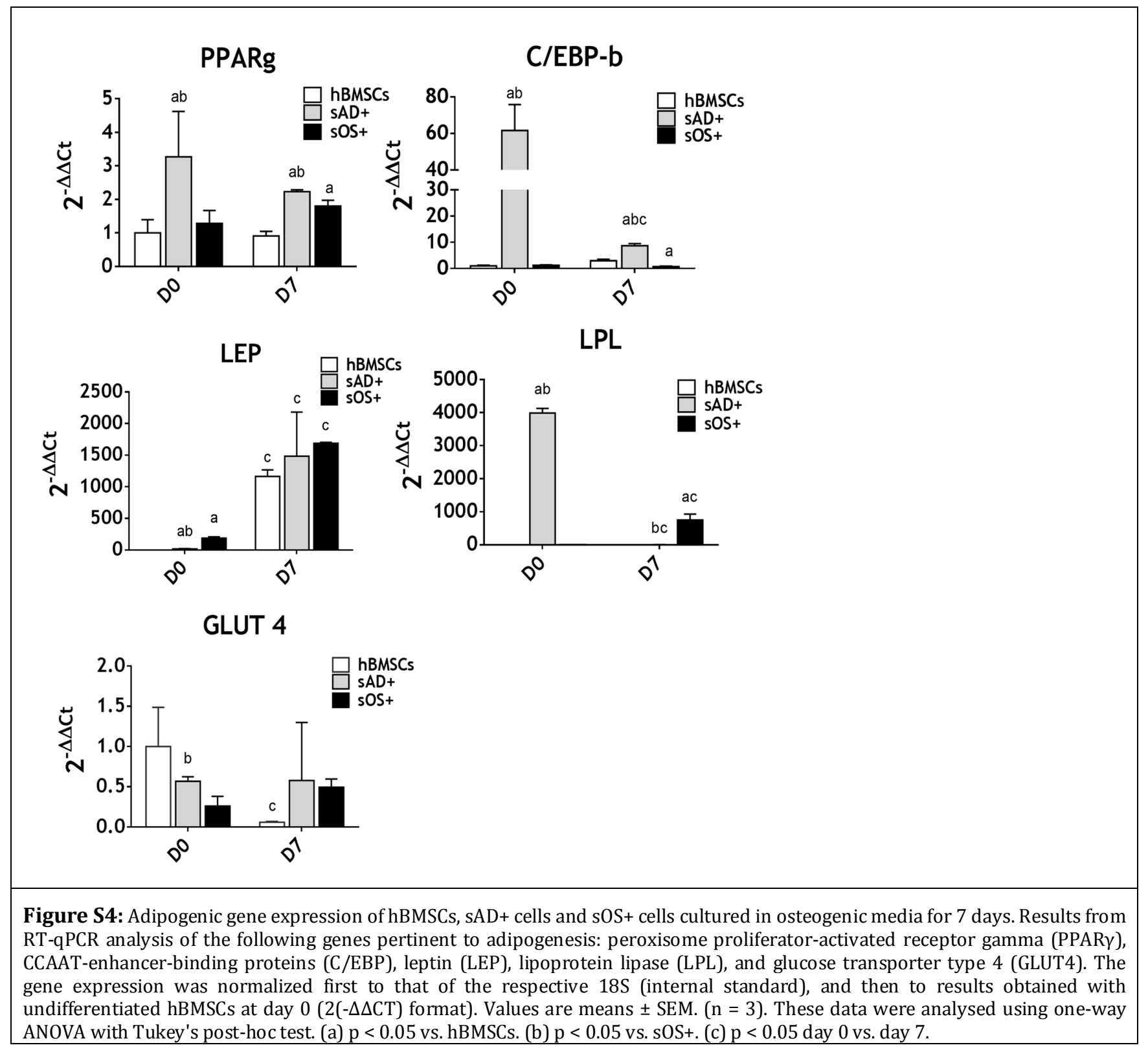



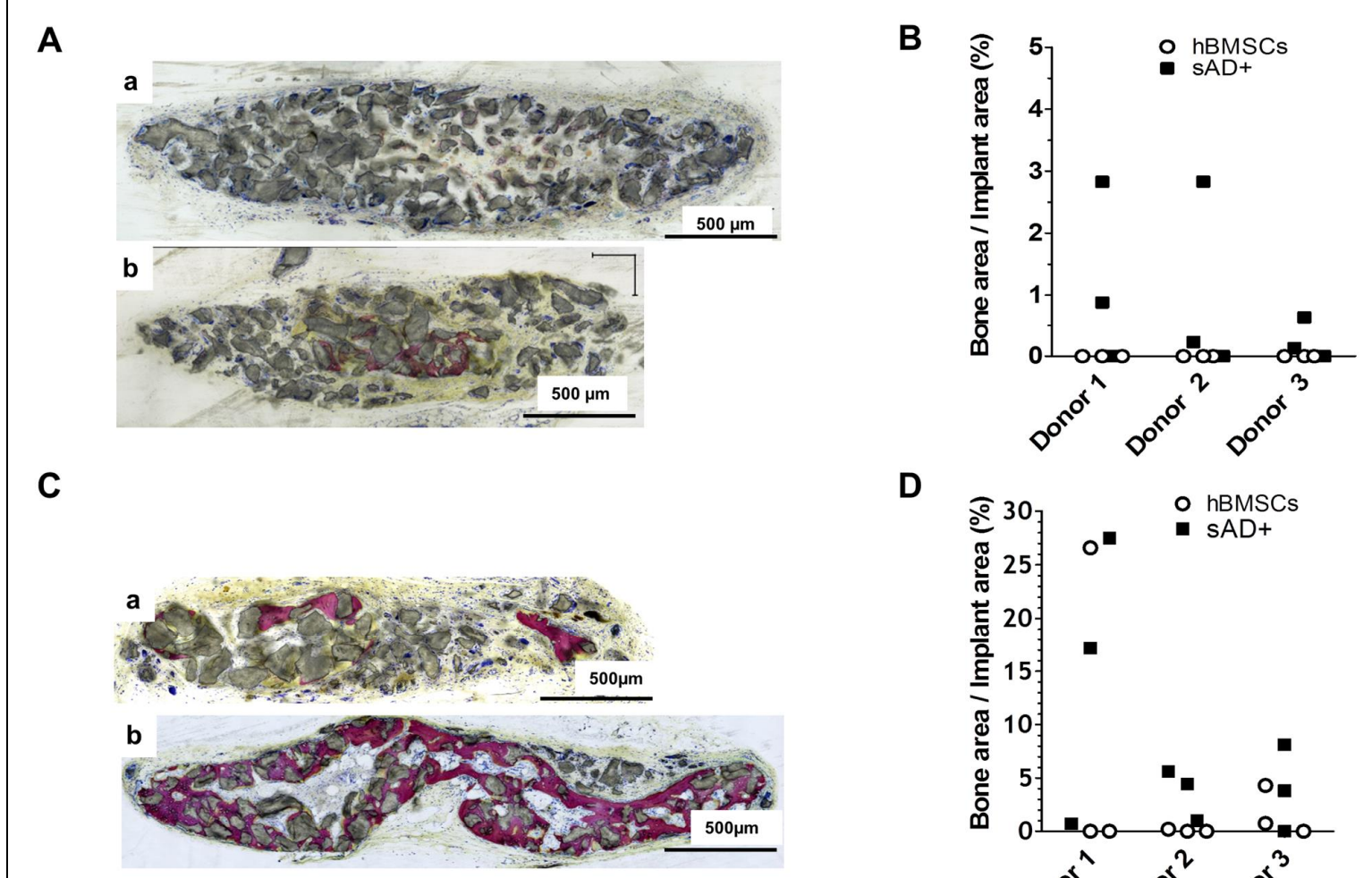

D

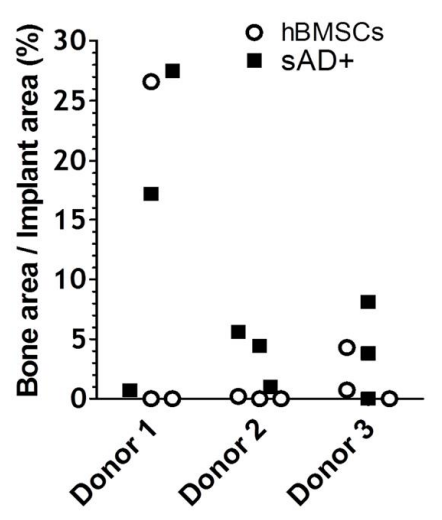

Figure S5: De novo bone formation in tissue engineered constructs containing either hBMSCs or sAD+ cells from 3 separate donors. Representative histology sections of explanted constructs stained with Picrofuchsin /Stevelen\&s blue that were seeded with either hBMSCs (frames $\mathrm{Aa}$ and $\mathrm{Ca}$ ) or sAD+ cells (frames $\mathrm{Ab}$ and $\mathrm{Cb}$ ) from 3 separate donors on coral particles and ectopically implanted for either 6 (frames $\mathrm{Aa}-\mathrm{Ab}$ ) or 10 weeks (frames $\mathrm{Ca}-\mathrm{Cb}$ ) in nude mice. Quantification of the total bone area was performed at 6 weeks (frame B) and 10 weeks (frame D) post-implantation. The results are presented in a single plot format and are expressed as a percentage of the Bone area / Total implant area (\%) $(n=3$ for each donor). 
Moya et al.
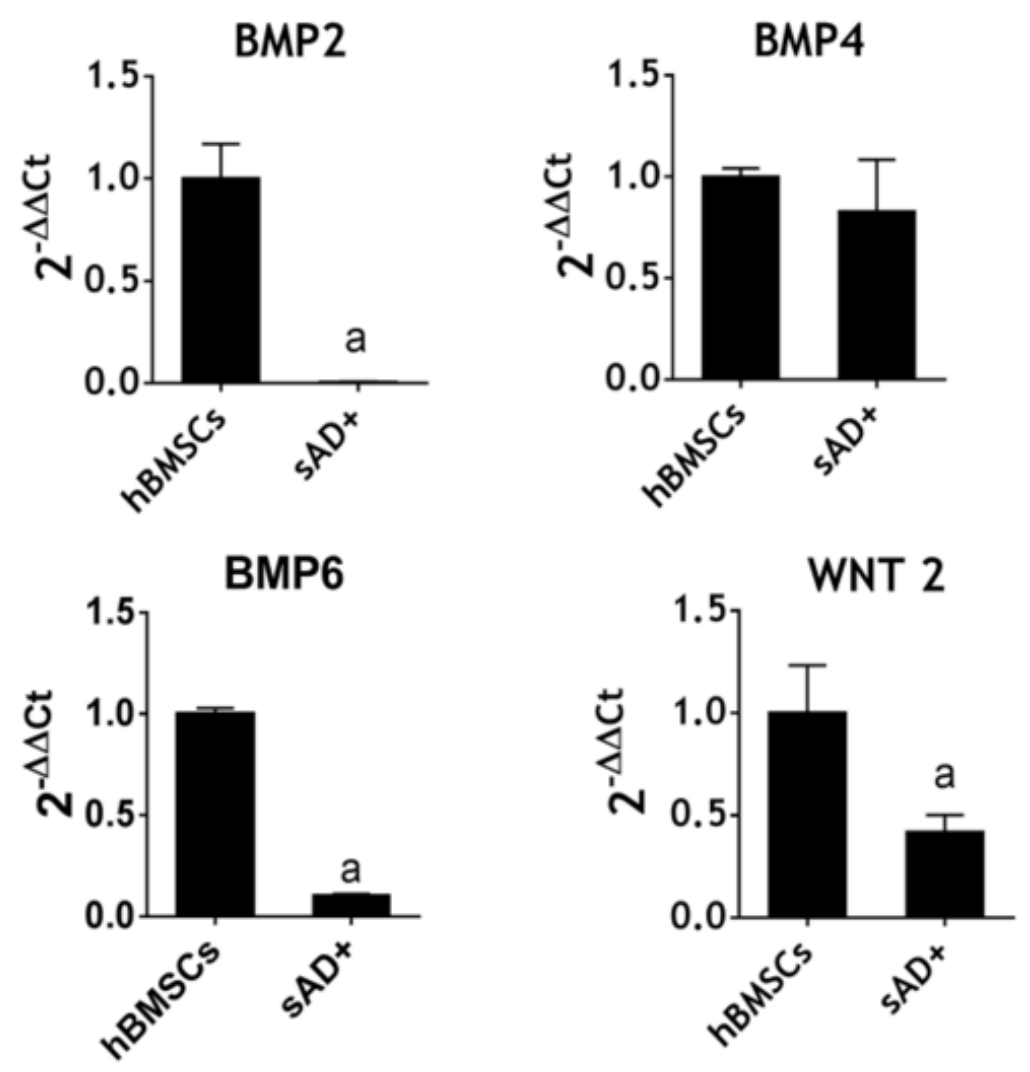

Figure S6: BMPs and Wnt Gene expression of hBMSCs and sAD+ cells. Results from RT-qPCR analysis of the following genes: Bone morphogenetic 2 (BMP2), Bone morphogenetic 4 (BMP4), Bone morphogen etic 6 (BMP6) and Wingless-type MMTV in tegration site 2 (Wnt2). The gene expression was normalized first to that of the respective 18S (internal standard), and then to results obtained with undifferentiated hBMSCs $(2(-\Delta \Delta C \mathrm{CT})$ format). Values are means \pm SEM. $(\mathrm{n}=3)$. These data were analyzed using Mann-Whitney test. (a) $\mathrm{p}<0.05$ vs. hBMSCs. 
Moya et al.

Table S1. 1. List of TaqMan Gene Expression Assays used in RT-PCR analysis

\begin{tabular}{|c|c|c|}
\hline Gene symbol & Name & Assay ID * \\
\hline PPAR $\gamma$ & Peroxisome proliferator-activated receptor gamma & Hs01115513_m1 \\
\hline CEBPA & CCAAT-enhancer-binding proteins & Hs00269972_s1 \\
\hline LEP & Leptin & Hs00174877_m1 \\
\hline LPL & Lipoprotein lipase & Hs00173425_m1 \\
\hline GLUT 4 / SLC2A4 & Glucose transporter type 4 & Hs00168966_m1 \\
\hline ALP & Alkaline phosphatase & Hs01029144_m1 \\
\hline RUNX 2 & Runt-related transcription factor 2 & Hs00231692_m1 \\
\hline IBSP & Integrin binding sialoprotein & Hs00173720_m1 \\
\hline bGLAP & Bone gamma-carboxyglutamate (Gla) protein & Hs00609452_g1 \\
\hline BMP2 & Bone morphogenetic 2 & Hs00154192_m1 \\
\hline BMP4 & Bone morphogenetic 4 & Hs00370078_m1 \\
\hline BMP6 & Bone morphogenetic 6 & Hs01099594_m1 \\
\hline WNT2 & Wingless-type MMTV integration site 2 & Hs00608224_m1 \\
\hline $18 \mathrm{~S}$ & 18S ribosomal RNA & Hs99999901_s1 \\
\hline
\end{tabular}

